TRANSACTIONS OF THE

AMERICAN MATHEMATICAL SOCIETY

Volume 355 , Number 7 , Pages 2925-2948

S 0002-9947(03)03256-2

Article electronically published on February 25, 2003

\title{
INTEGRATION BY PARTS FORMULAS INVOLVING GENERALIZED FOURIER-FEYNMAN TRANSFORMS ON FUNCTION SPACE
}

\author{
SEUNG JUN CHANG, JAE GIL CHOI, AND DAVID SKOUG
}

\begin{abstract}
In an upcoming paper, Chang and Skoug used a generalized Brownian motion process to define a generalized analytic Feynman integral and a generalized analytic Fourier-Feynman transform. In this paper we establish several integration by parts formulas involving generalized Feynman integrals, generalized Fourier-Feynman transforms, and the first variation of functionals of the form $F(x)=f\left(\left\langle\alpha_{1}, x\right\rangle, \ldots,\left\langle\alpha_{n}, x\right\rangle\right)$ where $\langle\alpha, x\rangle$ denotes the PaleyWiener-Zygmund stochastic integral $\int_{0}^{T} \alpha(t) d x(t)$.
\end{abstract}

\section{INTRODUCTION}

In [11, Park and Skoug, working in the setting of one-parameter Wiener space $C_{0}[0, T]$ established several integration by parts formulas involving analytic Feynman integrals, Fourier-Feynman transforms, and the first variation of functionals of the form

$$
F(x)=f\left(\left\langle\alpha_{1}, x\right\rangle, \cdots,\left\langle\alpha_{n}, x\right\rangle\right)
$$

where $\langle\alpha, x\rangle$ denotes the Paley-Wiener-Zygmund stochastic integral $\int_{0}^{T} \alpha(t) d x(t)$.

In this paper, we also study functionals of the form (1.1) but with $x$ in a very general function space $C_{a, b}[0, T]$ rather than in the Wiener space $C_{0}[0, T]$. The Wiener process used in [1] is free of drift and is stationary in time while the stochastic process used in this paper is nonstationary in time, is subject to a drift $a(t)$, and can be used to explain the position of the Ornstein-Uhlenbeck process in an external force field 10. It turns out, as noted in Remark 3.1 below, that including a drift term $a(t)$ makes establishing various integration by parts formulas for Fourier-Feynman transforms very difficult.

By choosing $a(t)=0$ and $b(t)=t$ on $[0, T]$, the function space $C_{a, b}[0, T]$ reduces to the Wiener space $C_{0}[0, T]$, and so the results in [11] are immediate corollaries of the results in this paper. For related work see [3], [4], and [6].

Received by the editors September 6, 2002 and, in revised form, November 15, 2002

2000 Mathematics Subject Classification. Primary 60J65, 28C20.

Key words and phrases. Generalized Brownian motion process, generalized analytic Feynman integral, generalized analytic Fourier-Feynman transform, first variation, Cameron-Storvick type theorem.

The present research was conducted by the research fund of Dankook University in 2000.

(C)2003 American Mathematical Society 


\section{DEFINITIONS AND PRELIMINARIES}

In this section we list the appropriate preliminaries and definitions from [5] that are needed to establish our parts formulas in Sections 3, 4 and 5 below.

Let $D=[0, T]$ and let $(\Omega, \mathcal{B}, P)$ be a probability measure space. A real-valued stochastic process $Y$ on $(\boldsymbol{\Omega}, \mathcal{B}, P)$ and $D$ is called a generalized Brownian motion process if $Y(0, \omega)=0$ almost everywhere and for $0=t_{0}<t_{1}<\cdots<t_{n} \leq T$, the $n$-dimensional random vector $\left(Y\left(t_{1}, \omega\right), \cdots, Y\left(t_{n}, \omega\right)\right)$ is normally distributed with the density function

$$
\begin{aligned}
K(\vec{t}, \vec{\eta}) & =\left((2 \pi)^{n} \prod_{j=1}^{n}\left(b\left(t_{j}\right)-b\left(t_{j-1}\right)\right)\right)^{-1 / 2} \\
& \cdot \exp \left\{-\frac{1}{2} \sum_{j=1}^{n} \frac{\left(\left(\eta_{j}-a\left(t_{j}\right)\right)-\left(\eta_{j-1}-a\left(t_{j-1}\right)\right)\right)^{2}}{b\left(t_{j}\right)-b\left(t_{j-1}\right)}\right\}
\end{aligned}
$$

where $\vec{\eta}=\left(\eta_{1}, \cdots, \eta_{n}\right), \eta_{0}=0, \vec{t}=\left(t_{1}, \cdots, t_{n}\right)$, $a(t)$ is an absolutely continuous real-valued function on $[0, T]$ with $a(0)=0, a^{\prime}(t) \in L^{2}[0, T]$, and $b(t)$ is a strictly increasing, continuously differentiable real-valued function with $b(0)=0$ and $b^{\prime}(t)>$ 0 for each $t \in[0, T]$.

As explained in [13, pp. 18-20], $Y$ induces a probability measure $\mu$ on the measurable space $\left(\mathbb{R}^{D}, \mathcal{B}^{D}\right)$ where $\mathbb{R}^{D}$ is the space of all real-valued functions $x(t), t \in D$, and $\mathcal{B}^{D}$ is the smallest $\sigma$-algebra of subsets of $\mathbb{R}^{D}$ with respect to which all the coordinate evaluation maps $e_{t}(x)=x(t)$ defined on $\mathbb{R}^{D}$ are measurable. The triple $\left(\mathbb{R}^{D}, \mathcal{B}^{D}, \mu\right)$ is a probability measure space. This measure space is called the function space induced by the generalized Brownian motion process $Y$ determined by $a(\cdot)$ and $b(\cdot)$.

We note that the generalized Brownian motion process $Y$ determined by $a(\cdot)$ and $b(\cdot)$ is a Gaussian process with mean function $a(t)$ and covariance function $r(s, t)=$ $\min \{b(s), b(t)\}$. By Theorem 14.2, [13. p. 187], the probability measure $\mu$ induced by $Y$, taking a separable version, is supported by $C_{a, b}[0, T]$ (which is equivalent to the Banach space of continuous functions $x$ on $[0, T]$ with $x(0)=0$ under the sup norm). Hence $\left(C_{a, b}[0, T], \mathcal{B}\left(C_{a, b}[0, T]\right), \mu\right)$ is the function space induced by $Y$ where $\mathcal{B}\left(C_{a, b}[0, T]\right)$ is the Borel $\sigma$-algebra of $C_{a, b}[0, T]$.

A subset $B$ of $C_{a, b}[0, T]$ is said to be scale-invariant measurable [9] provided $\rho B$ is $\mathcal{B}\left(C_{a, b}[0, T]\right)$-measurable for all $\rho>0$, and a scale-invariant measurable set $N$ is said to be a scale-invariant null set provided $\mu(\rho N)=0$ for all $\rho>0$. A property that holds except on a scale-invariant null set is said to hold scale-invariant almost everywhere (s-a.e.).

Let $L_{a, b}^{2}[0, T]$ be the Hilbert space of functions on $[0, T]$ that are Lebesgue measurable and square integrable with respect to the Lebesgue-Stieltjes measures on $[0, T]$ induced by $a(\cdot)$ and $b(\cdot)$; i.e.,

$$
L_{a, b}^{2}[0, T]=\left\{v: \int_{0}^{T} v^{2}(s) d b(s)<\infty \text { and } \int_{0}^{T} v^{2}(s) d|a|(s)<\infty\right\}
$$

where $|a|(t)$ denotes the total variation of the function $a$ on the interval $[0, t]$.

For $u, v \in L_{a, b}^{2}[0, T]$, let

$$
(u, v)_{a, b}=\int_{0}^{T} u(t) v(t) d[b(t)+|a|(t)] .
$$


Then $(\cdot, \cdot)_{a, b}$ is an inner product on $L_{a, b}^{2}[0, T]$ and $\|u\|_{a, b}=\sqrt{(u, u)_{a, b}}$ is a norm on $L_{a, b}^{2}[0, T]$. In particular, note that $\|u\|_{a, b}=0$ if and only if $u(t)=0$ a.e. on $[0, T]$. Furthermore, $\left(L_{a, b}^{2}[0, T],\|\cdot\|_{a, b}\right)$ is a separable Hilbert space.

Let $\left\{\phi_{j}\right\}_{j=1}^{\infty}$ be a complete orthogonal set of real-valued functions of bounded variation on $[0, T]$ such that

$$
\left(\phi_{j}, \phi_{k}\right)_{a, b}= \begin{cases}0, & j \neq k \\ 1, & j=k,\end{cases}
$$

and for each $v \in L_{a, b}^{2}[0, T]$, let

$$
v_{n}(t)=\sum_{j=1}^{n}\left(v, \phi_{j}\right)_{a, b} \phi_{j}(t)
$$

for $n=1,2, \cdots$. Then for each $v \in L_{a, b}^{2}[0, T]$, the Paley-Wiener-Zygmund (PWZ) stochastic integral $\langle v, x\rangle$ is defined by the formula

$$
\langle v, x\rangle=\lim _{n \rightarrow \infty} \int_{0}^{T} v_{n}(t) d x(t)
$$

for all $x \in C_{a, b}[0, T]$ for which the limit exists; one can show that for each $v \in$ $L_{a, b}^{2}[0, T]$, the PWZ integral $\langle v, x\rangle$ exists for $\mu$-a.e. $x \in C_{a, b}[0, T]$.

We denote the function space integral of a $\mathcal{B}\left(C_{a, b}[0, T]\right)$-measurable functional $F$ by

$$
E[F]=\int_{C_{a, b}[0, T]} F(x) d \mu(x)
$$

whenever the integral exists.

We are now ready to state the definition of the generalized analytic Feynman integral.

Definition 2.1. Let $\mathbb{C}$ denote the complex numbers. Let $\mathbb{C}_{+}=\{\lambda \in \mathbb{C}: \operatorname{Re} \lambda>0\}$ and $\widetilde{\mathbb{C}}_{+}=\{\lambda \in \mathbb{C}: \lambda \neq 0$ and $\operatorname{Re} \lambda \geq 0\}$. Let $F: C_{a, b}[0, T] \longrightarrow \mathbb{C}$ be such that for each $\lambda>0$, the function space integral

$$
J(\lambda)=\int_{C_{a, b}[0, T]} F\left(\lambda^{-\frac{1}{2}} x\right) d \mu(x)
$$

exists for all $\lambda>0$. If there exists a function $J^{*}(\lambda)$ analytic in $\mathbb{C}_{+}$such that $J^{*}(\lambda)=J(\lambda)$ for all $\lambda>0$, then $J^{*}(\lambda)$ is defined to be the analytic function space integral of $F$ over $C_{a, b}[0, T]$ with parameter $\lambda$, and for $\lambda \in \mathbb{C}_{+}$we write

$$
E^{\operatorname{an}_{\lambda}}[F] \equiv E_{x}^{\operatorname{an}_{\lambda}}[F(x)]=J^{*}(\lambda) .
$$

Let $q \neq 0$ be a real number and let $F$ be a functional such that $E^{\mathrm{an}_{\lambda}}[F]$ exists for all $\lambda \in \mathbb{C}_{+}$. If the following limit exists, we call it the generalized analytic Feynman integral of $F$ with parameter $q$ and we write

$$
E^{\operatorname{anf}_{q}}[F] \equiv E_{x}^{\operatorname{anf}_{q}}[F(x)]=\lim _{\lambda \rightarrow-i q} E^{\operatorname{an}_{\lambda}}[F]
$$

where $\lambda$ approaches $-i q$ through values in $\mathbb{C}_{+}$.

Next (see [5], [7], [1], [8], and [6]) we state the definition of the generalized analytic Fourier-Feynman transform (GFFT). 
Definition 2.2. For $\lambda \in \mathbb{C}_{+}$and $y \in C_{a, b}[0, T]$, let

$$
T_{\lambda}(F)(y)=E_{x}^{\mathrm{an}_{\lambda}}[F(y+x)] .
$$

For $p \in(1,2]$, we define the $L_{p}$ analytic $\operatorname{GFFT}, T_{q}(p ; F)$ of $F$, by the formula $\left(\lambda \in \mathbb{C}_{+}\right)$,

$$
T_{q}(p ; F)(y)=\lim _{\lambda \rightarrow-i q} T_{\lambda}(F)(y)
$$

if it exists; i.e., for each $\rho>0$,

$$
\lim _{\lambda \rightarrow-i q} \int_{C_{a, b}[0, T]}\left|T_{\lambda}(F)(\rho y)-T_{q}(p ; F)(\rho y)\right|^{p^{\prime}} d \mu(y)=0
$$

where $1 / p+1 / p^{\prime}=1$. We define the $L_{1}$ analytic GFFT, $T_{q}(1 ; F)$ of $F$, by the formula $\left(\lambda \in \mathbb{C}_{+}\right)$

$$
T_{q}(1 ; F)(y)=\lim _{\lambda \rightarrow-i q} T_{\lambda}(F)(y)
$$

if it exists.

We note that for $1 \leq p \leq 2, T_{q}(p ; F)$ is only defined as s-a.e. We also note that if $T_{q}(p ; F)$ exists and if $F \approx G$, then $T_{q}(p ; G)$ exists and $T_{q}(p ; G) \approx T_{q}(p ; F)$.

Next we give the definition of the first variation of a functional $F$ on $C_{a, b}[0, T]$ followed by a very fundamental Cameron-Storvick type theorem [2] which was established in [5, Theorem 3.5].

Definition 2.3. Let $F$ be a $\mathcal{B}\left(C_{a, b}[0, T]\right)$-measurable functional on $C_{a, b}[0, T]$ and let $w \in C_{a, b}[0, T]$. Then

$$
\delta F(x \mid w)=\left.\frac{\partial}{\partial h} F(x+h w)\right|_{h=0}
$$

(if it exists) is called the first variation of $F$.

Throughout this paper, when working with $\delta F(x \mid w)$, we will always require $w$ to be an element of $A$ where

$$
A=\left\{w \in C_{a, b}[0, T]: w(t)=\int_{0}^{t} z(s) d b(s) \text { for some } z \in L_{a, b}^{2}[0, T]\right\} .
$$

Note that for $F(x)$ of the form (1.1), $\delta F(x \mid w)$ acts like a directional derivative in the direction of $w$. For example, if $f\left(u_{1}, u_{2}\right)=\exp \left\{3 u_{1}+4 u_{2}\right\}$ and $F(x)=$ $f\left(\left\langle\alpha_{1}, x\right\rangle,\left\langle\alpha_{2}, x\right\rangle\right)$, then

$$
\begin{aligned}
\delta F(x \mid w) & =\left[3\left\langle\alpha_{1}, w\right\rangle+4\left\langle\alpha_{2}, w\right\rangle\right] \exp \left\{3\left\langle\alpha_{1}, x\right\rangle+4\left\langle\alpha_{2}, x\right\rangle\right\} \\
& =\left\langle\alpha_{1}, w\right\rangle f_{1}\left(\left\langle\alpha_{1}, x\right\rangle,\left\langle\alpha_{2}, x\right\rangle\right)+\left\langle\alpha_{2}, w\right\rangle f_{2}\left(\left\langle\alpha_{1}, x\right\rangle,\left\langle\alpha_{2}, x\right\rangle\right) .
\end{aligned}
$$

The following notation is used throughout the paper:

$$
\left(u, a^{\prime}\right)=\int_{0}^{T} u(t) a^{\prime}(t) d t=\int_{0}^{T} u(t) d a(t)
$$

and

$$
\left(u^{2}, b^{\prime}\right)=\int_{0}^{T} u^{2}(t) b^{\prime}(t) d t=\int_{0}^{T} u^{2}(t) d b(t)
$$

for $u \in L_{a, b}^{2}[0, T]$. Furthermore, for all $\lambda \in \tilde{\mathbb{C}}_{+}, \sqrt{\lambda}$ is always chosen to have positive real part. 
Theorem 2.1. Let $z \in L_{a, b}^{2}[0, T]$ be given and for $t \in[0, T]$, let $w(t)=\int_{0}^{t} z(s) d b(s)$. For each $\rho>0$, let $F(\rho x)$ be $\mu$-integrable on $C_{a, b}[0, T]$ and let $F(\rho x)$ have a first variation $\delta F(\rho x \mid \rho w)$ for all $x \in C_{a, b}[0, T]$ such that for some positive function $\eta(\rho)$,

$$
\sup _{|h| \leq \eta(\rho)}|\delta F(\rho x+\rho h w \mid \rho w)|
$$

is $\mu$-integrable. Then if any two of the three generalized analytic Feynman integrals in the following equation exist, then the third one also exists, and equality holds:

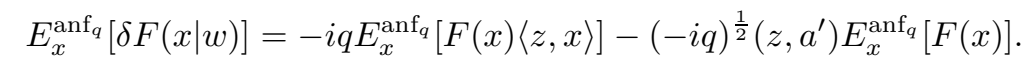

In fact, for each $\lambda \in \mathbb{C}_{+}$, the above conclusions also hold for analytic function space integrals,

$$
E_{x}^{\operatorname{an}_{\lambda}}[\delta F(x \mid w)]=\lambda E_{x}^{\operatorname{an}_{\lambda}}[F(x)\langle z, x\rangle]-\sqrt{\lambda}\left(z, a^{\prime}\right) E_{x}^{\mathrm{an}_{\lambda}}[F(x)]
$$

We finish this section by stating a very fundamental integration formula for the function space $C_{a, b}[0, T]$.

Let $\left\{\alpha_{1}, \cdots, \alpha_{n}\right\}$ be an orthonormal set of functions from $\left(L_{a, b}^{2}[0, T],\|\cdot\|_{a, b}\right)$, and for $j \in\{1, \cdots, n\}$ let

$$
A_{j} \equiv\left(\alpha_{j}, a^{\prime}\right)=\int_{0}^{T} \alpha_{j}(t) d a(t)
$$

and

$$
B_{j} \equiv\left(\alpha_{j}^{2}, b^{\prime}\right)=\int_{0}^{T} \alpha_{j}^{2}(t) d b(t) .
$$

Note that $B_{j}>0$ for each $j \in\{1,2, \cdots, n\}$, while for each $j, A_{j}$ may be positive, negative or zero. Then

Let $f: \mathbb{R}^{n} \rightarrow \mathbb{R}$ be Lebesgue measurable, and let $F(x)=f\left(\left\langle\alpha_{1}, x\right\rangle, \cdots,\left\langle\alpha_{n}, x\right\rangle\right)$.

$$
\begin{aligned}
& E[F] \equiv \int_{C_{a, b}[0, T]} f\left(\left\langle\alpha_{1}, x\right\rangle, \cdots,\left\langle\alpha_{n}, x\right\rangle\right) d \mu(x) \\
& =\left(\prod_{j=1}^{n} 2 \pi B_{j}\right)^{-\frac{1}{2}} \int_{\mathbb{R}^{n}} f\left(u_{1}, \cdots, u_{n}\right) \exp \left\{-\sum_{j=1}^{n} \frac{\left(u_{j}-A_{j}\right)^{2}}{2 B_{j}}\right\} d u_{1} \cdots d u_{n}
\end{aligned}
$$

in the sense that if either side exists, both sides exist and equality holds.

\section{INTEGRATION BY PARTS FORMULAS ON FUNCTION SPACE}

Let $n$ be a positive integer (fixed throughout this paper) and let $\left\{\alpha_{1}, \cdots, \alpha_{n}\right\}$ be an orthonormal set of functions from $\left(L_{a, b}^{2}[0, T],\|\cdot\|_{a, b}\right)$. Let $m$ be a nonnegative integer. Then for $1 \leq p<\infty$, let $\mathcal{B}(p ; m)$ be the space of all functionals of the form (1.1) for s-a.e. $x \in C_{a, b}[0, T]$ where all of the $k$ th-order partial derivatives $f_{j_{1}, \cdots, j_{k}}\left(u_{1}, \cdots, u_{n}\right)=f_{j_{1}, \cdots, j_{k}}(\vec{u})$ of $f: \mathbb{R}^{n} \rightarrow \mathbb{R}$ are continuous and in $L^{p}\left(\mathbb{R}^{n}\right)$ for $k \in\{0,1, \cdots, m\}$ and each $j_{i} \in\{1, \cdots, n\}$. Also, let $\mathcal{B}(\infty ; m)$ be the space of all functionals of the form (1.1) for s-a.e. $x \in C_{a, b}[0, T]$ where for $k=0,1, \cdots, m$, all of the $k$ th-order partial derivatives $f_{j_{1}, \cdots, j_{k}}(\vec{u})$ of $f$ are in $C_{0}\left(\mathbb{R}^{n}\right)$, the space of bounded continuous functions on $\mathbb{R}^{n}$ that vanish at infinity.

Our first lemma follows directly from the definitions of $\delta F(x \mid w)$ and $\mathcal{B}(p ; m)$. 
Lemma 3.1. Let $1 \leq p \leq \infty$ be given, let $m$ be a positive integer, let $F \in \mathcal{B}(p ; m)$ be given by equation (1.1) and let $w$ be an element of $A$. Then

$$
\delta F(x \mid w)=\sum_{j=1}^{n}\left\langle\alpha_{j}, w\right\rangle f_{j}\left(\left\langle\alpha_{1}, x\right\rangle, \cdots,\left\langle\alpha_{n}, x\right\rangle\right)
$$

for s-a.e. $x \in C_{a, b}[0, T]$. Furthermore, as a function of $x, \delta F(\cdot \mid w) \in \mathcal{B}(p ; m-1)$.

Lemma 3.2. Let $p, m$ and $F$ be as in Lemma 3.1. Let $z \in L_{a, b}^{2}[0, T]$ be given, and for $t \in[0, T]$, let $w(t)=\int_{0}^{t} z(s) d b(s)$. Let $G \in \mathcal{B}\left(p^{\prime} ; m\right)$ be given by

$$
G(x)=g\left(\left\langle\alpha_{1}, x\right\rangle, \cdots,\left\langle\alpha_{n}, x\right\rangle\right)
$$

for s-a.e. $x \in C_{a, b}[0, T]$. Define $R(x)=F(x) G(x)$ for $x \in C_{a, b}[0, T]$. Then $R \in \mathcal{B}(1 ; m)$, and as a function of $x, \delta R(\cdot \mid w) \in \mathcal{B}(1 ; m-1)$.

Proof. Let $r\left(u_{1}, \cdots, u_{n}\right)=f\left(u_{1}, \cdots, u_{n}\right) g\left(u_{1}, \cdots, u_{n}\right)$. Then $R(x)=r\left(\left\langle\alpha_{1}, x\right\rangle\right.$, $\left.\cdots,\left\langle\alpha_{n}, x\right\rangle\right)$ is an element of $\mathcal{B}(1 ; m)$ since all of the $k$ th-order partial derivatives of $r$ are continuous and in $L^{1}(\mathbb{R})$ for $k=0,1, \cdots, m$. Applying Lemma 3.1 we obtain that $\delta R(x \mid w)$, as a function of $x$, belongs to $\mathcal{B}(1 ; m-1)$.

Remark 3.1. Let $F, G$ and $R$ be as in Lemma 3.2 above. In evaluating $E\left[F\left(\lambda^{-\frac{1}{2}} x\right)\right]$, $E\left[R\left(\lambda^{-\frac{1}{2}} x\right)\right]$, and $E\left[\delta R\left(\lambda^{-\frac{1}{2}} x \mid w\right)\right]$ for $\lambda>0$, the expression

$$
H\left(\lambda ; u_{1}, \cdots, u_{n}\right) \equiv H(\lambda ; \vec{u})=\exp \left\{-\sum_{j=1}^{n} \frac{\left(\sqrt{\lambda} u_{j}-A_{j}\right)^{2}}{2 B_{j}}\right\}
$$

occurs, where $A_{j}$ and $B_{j}$ are given by equations (2.18) and (2.19) above. Clearly, for $\lambda>0,|H(\lambda ; \vec{u})| \leq 1$ for all $\vec{u} \in \mathbb{R}^{n}$ since $B_{j}>0$ for all $j=1, \cdots, n$. But for $\lambda \in \tilde{\mathbb{C}}_{+},|H(\lambda ; \vec{u})|$ is not necessarily bounded by 1 . Note that for each $\lambda \in \tilde{\mathbb{C}}_{+}$, $\sqrt{\lambda}=c+i d$ with $c \geq|d| \geq 0$. Hence, for each $\lambda \in \tilde{\mathbb{C}}_{+}$,

$$
\begin{aligned}
H(\lambda ; \vec{u}) & =\exp \left\{-\sum_{j=1}^{n} \frac{\left(\sqrt{\lambda} u_{j}-A_{j}\right)^{2}}{2 B_{j}}\right\} \\
& =\exp \left\{-\sum_{j=1}^{n} \frac{\left[\left(c^{2}-d^{2}+2 c d i\right) u_{j}^{2}-2(c+d i) A_{j} u_{j}+A_{j}^{2}\right]}{2 B_{j}}\right\},
\end{aligned}
$$

and so

$$
|H(\lambda ; \vec{u})|=\exp \left\{-\sum_{j=1}^{n} \frac{\left[\left(c^{2}-d^{2}\right) u_{j}^{2}-2 c A_{j} u_{j}+A_{j}^{2}\right]}{2 B_{j}}\right\} .
$$

Note that for $\lambda \in \mathbb{C}_{+}$, the case we consider throughout Section $3, \operatorname{Re}(\sqrt{\lambda})=$ $c>|d|=|\operatorname{Im}(\sqrt{\lambda})| \geq 0$, which implies that $c^{2}-d^{2}>0$. Hence, for each $\lambda \in \mathbb{C}_{+}$, $H(\lambda ; \vec{u})$, as a function of $\vec{u}$, is an element of $L^{p}\left(\mathbb{R}^{n}\right)$ for all $p \in[1,+\infty]$; in fact, $H(\lambda ; \vec{u})$ also belongs to $C_{0}\left(\mathbb{R}^{n}\right)$. These observations are critical to the development of the integration by parts formulas throughout Section 3.

In Sections 4 and 5 below we consider the case where $\lambda=-i q \in \tilde{\mathbb{C}}_{+}-\mathbb{C}_{+}$. In this case, $\sqrt{\lambda}=\sqrt{-i q}=c+i d$ with $c=\sqrt{|q| / 2}=|d|$. Hence, for $\lambda=-i q, q \in \mathbb{R}-\{0\}$, $c^{2}-d^{2}=0$, and so

$$
|H(-i q ; \vec{u})|=\exp \left\{\sum_{j=1}^{n} \frac{\left[\sqrt{2|q|} A_{j} u_{j}-A_{j}^{2}\right]}{2 B_{j}}\right\},
$$


which is not necessarily in $L^{p}\left(\mathbb{R}^{n}\right)$ for any $p \in[1,+\infty]$. Thus, in Sections 4 and 5 we will need to put additional restrictions on the functionals $F$ and $G$ in order to obtain the corresponding parts formulas involving Fourier-Feynman transforms.

Remark 3.2. Note that in the setting of [11, $a(t)=0$ and $b(t)=t$ on $[0, T]$ and so $A_{j}=\left(\alpha_{j}, a^{\prime}\right)=0$ and $B_{j}=\left(\alpha_{j}^{2}, b^{\prime}\right)=1$ for all $j \in\{1,2, \cdots, n\}$. Hence, for all $\lambda \in \tilde{\mathbb{C}}_{+}$,

$$
|H(\lambda ; \vec{u})|=\left|\exp \left\{-\frac{\lambda}{2} \sum_{j=1}^{n} u_{j}^{2}\right\}\right|=\exp \left\{-\frac{\operatorname{Re}(\lambda)}{2} \sum_{j=1}^{n} u_{j}^{2}\right\} \leq 1 .
$$

Theorem 3.3. Let $z \in L_{a, b}^{2}[0, T]$ be given and for $t \in[0, T]$, let $w(t)=\int_{0}^{t} z(s) d b(s)$. Let $p, m, F$ and $G$ be as in Lemma 3.2. Then for all $\lambda \in \mathbf{C}_{+}$,

$$
\begin{aligned}
& E_{x}^{\mathrm{an}_{\lambda}}[F(x) \delta G(x \mid w)+\delta F(x \mid w) G(x)] \\
& =\lambda E_{x}^{\mathrm{an}_{\lambda}}[F(x) G(x)\langle z, x\rangle]-\sqrt{\lambda}\left(z, a^{\prime}\right) E_{x}^{\mathrm{an}_{\lambda}}[F(x) G(x)]
\end{aligned}
$$

where $\sqrt{\lambda}$ is chosen to have positive real part.

Proof. First define $R(x)=F(x) G(x)$ and let

$$
r\left(u_{1}, \cdots, u_{n}\right)=f\left(u_{1}, \cdots, u_{n}\right) g\left(u_{1}, \cdots, u_{n}\right) .
$$

Then by Lemma 3.2, $R \in \mathcal{B}(1 ; m)$ and $\delta R(\cdot \mid w) \in \mathcal{B}(1 ; m-1)$. Furthermore, all of the $k$ th-order partial derivatives of $r$ are continuous and in $L^{1}\left(\mathbb{R}^{n}\right)$ for $k=0,1, \cdots, m$. Hence, $R(\rho x)$ is $\mu$-integrable on $C_{a, b}[0, T]$ for each $\rho>0$. In addition, for s-a.e. $x \in C_{a, b}[0, T]$,

$$
\begin{aligned}
\delta R(x \mid w)= & F(x) \delta G(x \mid w)+\delta F(x \mid w) G(x) \\
= & f\left(\left\langle\alpha_{1}, x\right\rangle, \cdots,\left\langle\alpha_{n}, x\right\rangle\right) \sum_{j=1}^{n}\left\langle\alpha_{j}, w\right\rangle g_{j}\left(\left\langle\alpha_{1}, x\right\rangle, \cdots,\left\langle\alpha_{n}, x\right\rangle\right) \\
& \quad+g\left(\left\langle\alpha_{1}, x\right\rangle, \cdots,\left\langle\alpha_{n}, x\right\rangle\right) \sum_{j=1}^{n}\left\langle\alpha_{j}, w\right\rangle f_{j}\left(\left\langle\alpha_{1}, x\right\rangle, \cdots,\left\langle\alpha_{n}, x\right\rangle\right) .
\end{aligned}
$$

But for all $u \in L_{a, b}^{2}[0, T]$,

$$
\begin{aligned}
|\langle u, w\rangle| & =\left|\int_{0}^{T} u(s) d w(s)\right| \\
& =\left|\int_{0}^{T} u(s) z(s) d b(s)\right| \\
& \leq \int_{0}^{T}|u(s) z(s)| d[b(s)+|a|(s)] \\
& =(|u|,|z|)_{a, b} \\
& \leq\|u\|_{a, b}\|z\|_{a, b} .
\end{aligned}
$$

In particular, since $\left\{\alpha_{1}, \cdots, \alpha_{n}\right\}$ are orthonormal, $\left|\left\langle\alpha_{j}, w\right\rangle\right| \leq\|z\|_{a, b}$ for each $j \in$ $\{1,2, \cdots, n\}$. 
Next, using (3.8) and (3.9), we see that for $\rho>0$ and $h>0$,

$$
\begin{aligned}
& |\delta R(\rho x+\rho h w \mid \rho w)| \\
& \leq \rho\|z\|_{a, b}\left|f\left(\left\langle\alpha_{1}, \rho x+\rho h w\right\rangle, \cdots,\left\langle\alpha_{n}, \rho x+\rho h w\right\rangle\right)\right| \\
& \cdot \sum_{j=1}^{n}\left|g_{j}\left(\left\langle\alpha_{1}, \rho x+\rho h w\right\rangle, \cdots,\left\langle\alpha_{n}, \rho x+\rho h w\right\rangle\right)\right| \\
& +\rho\|z\|_{a, b}\left|g\left(\left\langle\alpha_{1}, \rho x+\rho h w\right\rangle, \cdots,\left\langle\alpha_{n}, \rho x+\rho h w\right\rangle\right)\right| \\
& \cdot \sum_{j=1}^{n}\left|f_{j}\left(\left\langle\alpha_{1}, \rho x+\rho h w\right\rangle, \cdots,\left\langle\alpha_{n}, \rho x+\rho h w\right\rangle\right)\right| .
\end{aligned}
$$

But this implies that $\delta R(\rho x+\rho h w \mid \rho w)$, as a function of $x$, is $\mu$-integrable for all $\rho>0$ and $h>0$. This can be seen by integrating the right-hand side of (3.10) term by term. For example, using $(2.20)$, we see that for any $l \in\{1, \cdots, n\}$,

$$
\begin{aligned}
& E\left[\left|f\left(\left\langle\alpha_{1}, \rho x+\rho h w\right\rangle, \cdots,\left\langle\alpha_{n}, \rho x+\rho h w\right\rangle\right)\right|\right. \\
& \left.\cdot\left|g_{l}\left(\left\langle\alpha_{1}, \rho x+\rho h w\right\rangle, \cdots,\left\langle\alpha_{n}, \rho x+\rho h w\right\rangle\right)\right|\right] \\
& =\left(\prod_{j=1}^{n} 2 \pi \rho^{2} B_{j}\right)^{-1 / 2} \int_{\mathbb{R}^{n}}\left|f\left(u_{1}, \cdots, u_{n}\right) g_{l}\left(u_{1}, \cdots, u_{n}\right)\right| \\
& \cdot \exp \left\{-\sum_{j=1}^{n} \frac{\left[u_{j}-\rho\left(A_{j}+h\left\langle\alpha_{j}, w\right\rangle\right)\right]^{2}}{2 \rho^{2} B_{j}}\right\} d u_{1} \cdots d u_{n} \\
& \leq\left(\prod_{j=1}^{n} 2 \pi \rho^{2} B_{j}\right)^{-1 / 2}\|f\|_{p}\left\|g_{l}\right\|_{p^{\prime}}<\infty .
\end{aligned}
$$

Thus, using (3.10) and (3.11), we obtain that for $\rho>0$ and $h>0$,

$$
\begin{aligned}
& E[|\delta R(\rho x+\rho h w \mid \rho w)|] \\
& \quad \leq \rho\|z\|_{a, b}\left(\prod_{j=1}^{n} 2 \pi \rho^{2} B_{j}\right)^{-1 / 2}\left[\|f\|_{p} \sum_{l=1}^{n}\left\|g_{l}\right\|_{p^{\prime}}+\|g\|_{p^{\prime}} \sum_{l=1}^{n}\left\|f_{l}\right\|_{p}\right]<\infty .
\end{aligned}
$$

Next, using (3.8), (2.19), (3.3), and (3.4), we see that for all $\lambda>0$,

$$
\begin{aligned}
& E\left[F\left(\lambda^{-\frac{1}{2}} x\right) \delta G\left(\lambda^{-\frac{1}{2}} x \mid w\right)+\delta F\left(\lambda^{-\frac{1}{2}} x \mid w\right) G(x)\right] \\
& =\left(\prod_{j=1}^{n} \frac{\lambda}{2 \pi B_{j}}\right)^{1 / 2} \int_{\mathbb{R}^{n}}\left[f(\vec{u}) \sum_{l=1}^{n}\left\langle\alpha_{l}, w\right\rangle g_{l}(\vec{u})\right. \\
& \left.+g(\vec{u}) \sum_{l=1}^{n}\left\langle\alpha_{l}, w\right\rangle f_{l}(\vec{u})\right] H(\lambda ; \vec{u}) d \vec{u} .
\end{aligned}
$$

But, as noted in Remark 3.1 above, for each $\lambda \in \mathbf{C}_{+}, H(\lambda ; \vec{u})$ is an element of $C_{0}\left(\mathbb{R}^{n}\right)$, and so the integrand on the right-hand side of $(3.12)$ is in $L^{1}\left(\mathbb{R}^{n}\right)$. Hence,

$$
E_{x}^{\operatorname{an}_{\lambda}}[\delta R(x \mid w)]=E_{x}^{\operatorname{an}_{\lambda}}[F(x) \delta G(x \mid w)+\delta F(x \mid w) G(x)]
$$

exists for all $\lambda \in \mathbf{C}_{+}$. A similar argument shows that the analytic function space integral $E_{x}^{a_{\lambda}}[F(x) G(x)]$ also exists for all $\lambda \in \mathbf{C}_{+}$. Equation (3.7) now follows from Theorem 2.1 above; in particular, from equation (2.17) with $F(x)$ replaced with $R(x)$. 
The following two corollaries are special cases of Theorem 3.3.

Corollary 3.4. Let $z, w$, and $m$ be as in Theorem 3.3. Let $F \in \mathcal{B}(2 ; m)$ be given by (1.1). Then for all $\lambda \in \mathbb{C}_{+}$,

$$
\begin{aligned}
& E_{x}^{\mathrm{an}_{\lambda}[F(x) \delta F(x \mid w)]} \\
& \quad=\frac{\lambda}{2} E_{x}^{\mathrm{an}_{\lambda}}\left[(F(x))^{2}\langle z, x\rangle\right]-\frac{\sqrt{\lambda}}{2}\left(z, a^{\prime}\right) E_{x}^{\mathrm{an}_{\lambda}}\left[(F(x))^{2}\right] .
\end{aligned}
$$

Proof. In Theorem 3.3, choose $p=2$ and $G(x)=F(x)$.

Corollary 3.5. Let $z_{1}$ and $z_{2}$ be elements of $L_{a, b}^{2}[0, T]$, and for $t \in[0, T]$, let $w_{j}(t)=\int_{0}^{t} z_{j}(s) d b(s)$ for $j \in\{1,2\}$. Let $F \in \mathcal{B}(2 ; m)$ be given by equation (1.1). Then for all $\lambda \in \mathbb{C}_{+}$,

$$
\begin{aligned}
E_{x}^{\mathrm{an}_{\lambda}} & {\left[F(x) \delta^{2} F\left(\cdot \mid w_{1}\right)\left(x \mid w_{2}\right)+\delta F\left(x \mid w_{2}\right) \delta F\left(x \mid w_{1}\right)\right] } \\
& =\lambda E_{x}^{\mathrm{an}_{\lambda}}\left[F(x) \delta F\left(x \mid w_{1}\right)\left\langle z_{2}, x\right\rangle\right]-\sqrt{\lambda}\left(z_{2}, a^{\prime}\right) E_{x}^{\mathrm{an}_{\lambda}}\left[F(x) \delta F\left(x \mid w_{1}\right)\right] .
\end{aligned}
$$

Proof. Let $p=2$ and $G(x)=\delta F\left(x \mid w_{1}\right)$ in Theorem 3.3.

Lemma 3.6. Let $p, m$ and $F$ be as in Lemma 3.1 above. Then for all $\lambda \in \mathbb{C}_{+}$,

$$
T_{\lambda}(F)(y)=E_{x}^{\mathrm{an}_{\lambda}}[F(y+x)]=\phi_{0}\left(\lambda ;\left\langle\alpha_{1}, y\right\rangle, \cdots,\left\langle\alpha_{n}, y\right\rangle\right)
$$

for s-a.e. $y \in C_{a, b}[0, T]$ where

$$
\phi_{0}\left(\lambda ; \xi_{1}, \cdots, \xi_{n}\right)=\left(\prod_{j=1}^{n} \frac{\lambda}{2 \pi B_{j}}\right)^{1 / 2} \int_{\mathbb{R}^{n}} f(\vec{u}+\vec{\xi}) H(\lambda ; \vec{u}) d \vec{u}
$$

with $B_{j}$ and $H$ given by equations (2.19) and (3.4) respectively.

Proof. For $\lambda>0$, equation (3.15) follows easily from equation (2.20). But for each $\lambda \in \mathbb{C}_{+}$, as shown in Remark 3.1 above, $H\left(\lambda ; u_{1}, \cdots, u_{n}\right)$ is an element of $L^{p}\left(\mathbb{R}^{n}\right) \cap C_{0}\left(\mathbb{R}^{n}\right)$ for all $p \in[1, \infty]$. Hence, for each $\lambda \in \mathbb{C}_{+}$and s-a.e. $y \in C_{a, b}[0, T]$,

$$
f\left(u_{1}+\left\langle\alpha_{1}, y\right\rangle, \cdots, u_{n}+\left\langle\alpha_{n}, y\right\rangle\right) H\left(\lambda ; u_{1}, \cdots, u_{n}\right)
$$

belongs to $L^{1}\left(\mathbb{R}^{n}\right)$ and so equation $(3.15)$ holds throughout $\mathbb{C}_{+}$.

Our next lemma follows from standard results for convolution products. The key is that for each $\lambda \in \mathbb{C}_{+}, H(\lambda ; \vec{u})$ is an element of $L^{p}\left(\mathbb{R}^{n}\right) \cap C_{0}\left(\mathbb{R}^{n}\right)$ for all $1 \leq p \leq+\infty$.

Lemma 3.7. Let $\phi_{0}$ be given by equation (3.16) above.

(a) If $f \in L^{1}\left(\mathbb{R}^{n}\right)$, then $\phi_{0}(\lambda ; \cdot) \in C_{0}\left(\mathbb{R}^{n}\right)$ for all $\lambda \in \mathbb{C}_{+}$.

(b) If $f \in L^{p}\left(\mathbb{R}^{n}\right)$ for some $p \in(1, \infty)$, then $\phi_{0}(\lambda ; \cdot) \in L^{p^{\prime}}\left(\mathbb{R}^{n}\right)$ for all $\lambda \in \mathbb{C}_{+}$ where $p^{\prime}=\frac{p}{p-1}$.

(c) If $f \in C_{0}\left(\mathbb{R}^{n}\right)$, then $\phi_{0}(\lambda ; \cdot) \in L^{1}\left(\mathbb{R}^{n}\right)$ for all $\lambda \in \mathbb{C}_{+}$.

Our next theorem follows immediately from Lemma 3.7.

Theorem 3.8. Let $1 \leq p \leq \infty$ be given. If $F \in \mathcal{B}(p ; m)$, then $T_{\lambda}(F) \in \mathcal{B}\left(p^{\prime} ; m\right)$. 
Theorem 3.9. Let $1 \leq p \leq \infty$ and $w \in A$ be given. Let $F \in \mathcal{B}(p ; m)$ be given by equation (1.1). Then for all $\lambda \in \mathbb{C}_{+}$and s-a.e. $y \in C_{a, b}[0, T]$,

$$
\begin{aligned}
\delta T_{\lambda}(F) & (y \mid w) \\
= & \left(\prod_{j=1}^{n} \frac{\lambda}{2 \pi B_{j}}\right)^{1 / 2} \int_{\mathbb{R}^{n}} \sum_{l=1}^{n}\left\langle\alpha_{l}, w\right\rangle f_{l}\left(u_{1}+\left\langle\alpha_{1}, y\right\rangle, \cdots, u_{n}+\left\langle\alpha_{n}, y\right\rangle\right) \\
& \cdot H\left(\lambda ; u_{1}, \cdots, u_{n}\right) d u_{1} \cdots d u_{n} \\
= & T_{\lambda}(\delta F(\cdot \mid w))(y),
\end{aligned}
$$

which, as a function of $y$, is an element of $\mathcal{B}\left(p^{\prime} ; m-1\right)$.

Proof. The fact that $\delta T_{\lambda}(F)(y \mid w)$ is an element of $\mathcal{B}\left(p^{\prime} ; m-1\right)$ follows directly from Theorem 3.8 and Lemma 3.1. To establish equation (3.17) for $\lambda>0$, simply calculate $\delta T_{\lambda}(F)(y \mid w)$ using equation (3.15), and then calculate $T_{\lambda}(\delta F(\cdot \mid w))(y)$ using equations (3.1) and (2.9). Finally, equation (3.17) holds throughout $\mathbb{C}_{+}$by analytic continuation in $\lambda$.

In our next theorem we obtain an integration by parts formula involving $T_{\lambda}(F)$ and $T_{\lambda}(G)$.

Theorem 3.10. Let $p, m, z, w, F$ and $G$ be as in Theorem 3.3. Then for all $\lambda \in \mathbb{C}_{+}$,

$$
\begin{aligned}
E_{x}^{\mathrm{an}_{\lambda}} & {\left[T_{\lambda}(F)(x) \delta T_{\lambda}(G)(x \mid w)+\delta T_{\lambda}(F)(x \mid w) T_{\lambda}(G)(x)\right] } \\
& =\lambda E_{\lambda}^{\mathrm{an}_{\lambda}}\left[T_{\lambda}(F)(x) T_{\lambda}(G)(x)\langle z, x\rangle\right]-\sqrt{\lambda}\left(z, a^{\prime}\right) E_{x}^{\mathrm{an}_{\lambda}}\left[T_{\lambda}(F)(x) T_{\lambda}(G)(x)\right] .
\end{aligned}
$$

Proof. For $x \in C_{a, b}[0, T]$, let $R(x)=T_{\lambda}(F)(x) T_{\lambda}(G)(x)$. Then by Theorem 3.8, $T_{\lambda}(F) \in \mathcal{B}\left(p^{\prime} ; m\right)$ and $T_{\lambda}(G) \in \mathcal{B}(p ; m)$. Hence, by Lemma $3.2, R$ belongs to $\mathcal{B}(1 ; m)$, and so by Lemma $3.1, \delta R(x \mid w)$, as a function of $x$, belongs to $\mathcal{B}(1 ; m-1)$. Thus, equation (3.18) follows from Theorem 3.3 with $F$ and $G$ replaced by $T_{\lambda}(F)$ and $T_{\lambda}(G)$ respectively.

Theorem 3.11. Let $m, z$ and $w$ be as in Lemma 3.2. Let $p \in[1,2]$ and let $F$ and $G$ in $\mathcal{B}(p ; m)$ be given by equations (1.1) and (3.2) respectively. Then for all $\lambda \in \mathbb{C}_{+}$,

$$
\begin{aligned}
& E_{x}^{\operatorname{an}_{\lambda}}\left[F(x) \delta T_{\lambda}(G)(x \mid w)+\delta F(x \mid w) T_{\lambda}(G)(x)\right] \\
& =\lambda E_{x}^{\operatorname{an}_{\lambda}}\left[F(x) T_{\lambda}(G)(x)\langle z, x\rangle\right]-\sqrt{\lambda}\left(z, a^{\prime}\right) E_{x}^{\operatorname{an}_{\lambda}}\left[F(x) T_{\lambda}(G)(x)\right] .
\end{aligned}
$$

Proof. Let $R(x)=F(x) T_{\lambda}(G)(x)$ for $x \in C_{a, b}[0, T]$. By Theorem 3.8, $T_{\lambda}(G)$ is an element of $\mathcal{B}\left(p^{\prime} ; m\right)$ and hence by Lemma $3.2, R$ belongs to $\mathcal{B}(1 ; m)$. Hence, by Lemma 3.1, $\delta R(x \mid w)$, as a function of $x$, belongs to $\mathcal{B}(1 ; m-1)$. Thus, equation (3.19) follows from Theorem 3.3 with $G$ replaced by $T_{\lambda}(G)$.

Corollary 3.12. Let $m, z, w, p$ and $F$ be as in Theorem 3.11. Then for all $\lambda \in \mathbb{C}_{+}$,

$$
\begin{aligned}
& E_{x}^{\operatorname{an}_{\lambda}}\left[F(x) \delta T_{\lambda}(F)(x \mid w)+\delta F(x \mid w) T_{\lambda}(F)(x)\right] \\
& =\lambda E_{x}^{\operatorname{an}_{\lambda}}\left[F(x) T_{\lambda}(F)(x)\langle z, x\rangle\right]-\sqrt{\lambda}\left(z, a^{\prime}\right) E_{x}^{\operatorname{an}_{\lambda}}\left[F(x) T_{\lambda}(F)(x)\right] .
\end{aligned}
$$

Proof. Simply choose $G=F$ in Theorem 3.11. 
Corollary 3.13. Let $m, z$ and $w$ be as in Lemma 3.2. Let $F \in \mathcal{B}(2 ; m)$ be given by equation (1.1). Then for all $\lambda \in \mathbb{C}_{+}$,

$$
\begin{aligned}
& E_{x}^{\operatorname{an}_{\lambda}}\left[T_{\lambda}(F)(x) \delta T_{\lambda}(F)(x \mid w)\right] \\
& =\frac{\lambda}{2} E_{x}^{\operatorname{an}_{\lambda}}\left[\left(T_{\lambda}(F)(x)\right)^{2}\langle z, x\rangle\right]-\frac{\sqrt{\lambda}\left(z, a^{\prime}\right)}{2} E_{x}^{\operatorname{an}_{\lambda}}\left[\left(T_{\lambda}(F)(x)\right)^{2}\right] .
\end{aligned}
$$

Proof. Simply choose $p=2$ and $G=F$ in Theorem 3.10.

4. Parts formulas involving $T_{q}(1 ; F)$ And $T_{q}(1 ; G)$

In this section we obtain various integration by parts formulas involving the analytic GFFTs $T_{q}(1 ; F)$ and $T_{q}(1 ; G)$. In view of equation (3.6) above, we clearly need to impose additional restrictions on the functionals $F$ and $G$ than were needed throughout Section 3.

Fix $q \in \mathbb{R}-\{0\}$. Then as $\lambda \rightarrow-i q$ through values in $\mathbb{C}_{+}, c=\operatorname{Re}(\sqrt{\lambda}) \rightarrow \sqrt{|q| / 2}$ and $|d| \rightarrow \sqrt{|q| / 2}$ where $d=\operatorname{Im}(\sqrt{\lambda})$.

Next using equations (3.3) through (3.6) we see that for all $\lambda \in \tilde{\mathbb{C}}_{+}$with $c=$ $\operatorname{Re}(\sqrt{\lambda})<((1+|q|) / 2)^{\frac{1}{2}}$

$$
\begin{aligned}
|H(\lambda ; \vec{u})| & =\exp \left\{-\sum_{j=1}^{n} \frac{\left[\left(c^{2}-d^{2}\right) u_{j}^{2}-2 c A_{j} u_{j}+A_{j}^{2}\right]}{2 B_{j}}\right\} \\
& \leq \exp \left\{\sum_{j=1}^{n} \frac{c A_{j} u_{j}}{B_{j}}\right\} \\
& \leq \exp \left\{\left(\frac{1+|q|}{2}\right)^{\frac{1}{2}} \sum_{j=1}^{n} \frac{\left|A_{j} u_{j}\right|}{B_{j}}\right\} .
\end{aligned}
$$

In addition,

$$
\begin{aligned}
& \int_{\mathbb{R}^{n}}|f(\vec{\xi}+\vec{u}) H(\lambda ; \vec{u})| d \vec{u} \\
&=\int_{\mathbb{R}^{n}}|f(\vec{u}) H(\lambda ; \vec{u}-\vec{\xi})| d \vec{u} \\
& \leq \exp \left\{-\operatorname{Re}(\sqrt{\lambda}) \sum_{j=1}^{n} \frac{A_{j} \xi_{j}}{B_{j}}\right\} \\
& \cdot \int_{\mathbb{R}^{n}}|f(\vec{u})| \exp \left\{\left(\frac{1+|q|}{2}\right)^{\frac{1}{2}} \sum_{j=1}^{n} \frac{\left|A_{j} u_{j}\right|}{B_{j}}\right\} d \vec{u} .
\end{aligned}
$$

For $f \in L^{1}\left(\mathbb{R}^{n}\right)$ let

$$
\mathcal{F}(f)(\vec{\xi})=(2 \pi)^{-\frac{n}{2}} \int_{\mathbb{R}^{n}} f(\vec{u}) \exp \left\{i \sum_{j=1}^{n} u_{j} \xi_{j}\right\} d \vec{u}
$$

denote the Fourier transform of $f$.

Theorem 4.1. Let $q \in \mathbb{R}-\{0\}$ be given. Let $F \in \mathcal{B}(1 ; m)$ be given by equation (1.1) with

$$
\int_{\mathbb{R}^{n}}\left|f_{j_{1}, \cdots, j_{k}}(\vec{u})\right| \exp \left\{\left(\frac{1+|q|}{2}\right)^{\frac{1}{2}} \sum_{j=1}^{n} \frac{\left|A_{j} u_{j}\right|}{B_{j}}\right\} d \vec{u}<\infty
$$


for all $k \in\{0,1, \cdots, m\}$ and each $j_{i} \in\{1, \cdots, n\}$. Furthermore, assume that

$$
\exp \left\{-\sum_{j=1}^{n} \frac{\sqrt{2|q|} A_{j} \xi_{j}}{2 B_{j}}\right\} \mathcal{F}(f(\cdot) H(-i q ; \cdot))\left(-\frac{q \xi_{1}}{B_{1}}, \cdots,-\frac{q \xi_{n}}{B_{n}}\right)
$$

belongs to $C_{0}\left(\mathbb{R}^{n}\right)$. Then

$$
\phi_{0}(-i q ; \vec{\xi}) \equiv\left(\prod_{j=1}^{n} \frac{-i q}{2 \pi B_{j}}\right)^{\frac{1}{2}} \int_{\mathbb{R}^{n}} f(\vec{\xi}+\vec{u}) H(-i q ; \vec{u}) d \vec{u}
$$

is an element of $C_{0}\left(\mathbb{R}^{n}\right)$. Furthermore, the $L_{1}$ analytic $G F F T, T_{q}(1 ; F)$ exists as an element of $\mathcal{B}(\infty ; m)$ and for s-a.e. $y \in C_{a, b}[0, T]$ is given by the formula

$$
T_{q}(1 ; F)(y)=\phi_{0}\left(-i q ;\left\langle\alpha_{1}, y\right\rangle, \cdots,\left\langle\alpha_{n}, y\right\rangle\right) .
$$

Proof. By (4.1) and (4.4) we know that $f(\cdot) H(-i q ; \cdot) \in L^{1}\left(\mathbb{R}^{n}\right)$, and so its Fourier transform, $\mathcal{F}(f(\cdot) H(-i q ; \cdot))(\vec{\xi})$ exists and belongs to $C_{0}\left(\mathbb{R}^{n}\right)$. Furthermore, by equations (4.6) and (3.4) and the fact that $\sqrt{-i q}=c+d i=\sqrt{|q| / 2}+d i$, we obtain

$$
\begin{aligned}
\phi_{0}(-i q ; \vec{\xi}) & \left(\prod_{j=1}^{n}-\frac{i q}{2 \pi B_{j}}\right)^{\frac{1}{2}} \int_{\mathbb{R}^{n}} f(\vec{\xi}+\vec{u}) H(-i q ; \vec{u}) d \vec{u} \\
= & \left(\prod_{j=1}^{n}-\frac{i q}{2 \pi B_{j}}\right)^{\frac{1}{2}} \int_{\mathbb{R}^{n}} f(\vec{\xi}+\vec{u}) \exp \left\{-\sum_{j=1}^{n} \frac{\left[\sqrt{-i q} u_{j}-A_{j}\right]^{2}}{2 B_{j}}\right\} d \vec{u} \\
= & \left(\prod_{j=1}^{n}-\frac{i q}{2 \pi B_{j}}\right)^{\frac{1}{2}} \int_{\mathbb{R}^{n}} f(\vec{u}) \exp \left\{-\sum_{j=1}^{n} \frac{\left[\sqrt{-i q}\left(u_{j}-\xi_{j}\right)-A_{j}\right]^{2}}{2 B_{j}}\right\} d \vec{u} \\
= & \left(\prod_{j=1}^{n}-\frac{i q}{B_{j}}\right)^{\frac{1}{2}} \exp \left\{-\sum_{j=1}^{n} \frac{\left[2 \sqrt{-i q} A_{j} \xi_{j}-i q \xi_{j}^{2}\right]}{2 B_{j}}\right\} \\
= & \left(\prod_{j=1}^{n}-\frac{i q}{B_{j}}\right)^{\frac{1}{2}} \exp \left\{-\sum_{j=1}^{n} \frac{\left[2 \sqrt{-i q} A_{j} \xi_{j}-i q \xi_{j}^{2}\right]}{2 B_{j}}\right\} \\
= & \left.\left(\prod_{j=1}^{n}-\frac{i q}{B_{j}}\right)^{\frac{1}{2}} \exp \left\{i \sum_{j=1}^{n} \frac{\left[q \xi_{j}^{2}-2 d A_{j} \xi_{j}\right]}{2 B_{j}}\right\} u_{j} \xi_{j} B_{j}\right\} d \vec{u} \\
& \cdot \exp \left\{-\sum_{j=1}^{n} \frac{\sqrt{2} \mid q\left(q \mid A_{j} \xi_{j}\right.}{2 B_{j}}\right\} \mathcal{F}(f(\cdot) H(-i q ; \cdot))\left(-\frac{q \xi_{1}}{B_{1}}, \cdots,-\frac{q \xi_{n}}{B_{n}}\right) .
\end{aligned}
$$

By assumption (4.5), it follows that $\phi_{0}(-i q ; \vec{\xi})$ is an element of $C_{0}\left(\mathbb{R}^{n}\right)$.

Finally, by equations (2.11), (3.15), (3.16), (4.8) and the dominated convergence theorem (the use of which is justified by (4.2)), it follows that for s-a.e. $y \in$ 
$C_{a, b}[0, T]$,

$$
\begin{aligned}
& T_{q}(1 ; F)(y) \\
& =\lim _{\lambda \rightarrow-i q} T_{\lambda}(F)(y) \\
& =\lim _{\lambda \rightarrow-i q} \phi_{0}\left(\lambda ;\left\langle\alpha_{1}, y\right\rangle, \cdots,\left\langle\alpha_{n}, y\right\rangle\right) \\
(4.9) & =\lim _{\lambda \rightarrow-i q}\left(\prod_{j=1}^{n} \frac{\lambda}{2 \pi B_{j}}\right)^{1 / 2} \int_{\mathbb{R}^{n}} f\left(u_{1}+\left\langle\alpha_{1}, y\right\rangle, \cdots, u_{n}+\left\langle\alpha_{n}, y\right\rangle\right) H(\lambda ; \vec{u}) d \vec{u} \\
& =\left(\prod_{j=1}^{n}-\frac{i q}{2 \pi B_{j}}\right)^{1 / 2} \int_{\mathbb{R}^{n}} f\left(u_{1}+\left\langle\alpha_{1}, y\right\rangle, \cdots, u_{n}+\left\langle\alpha_{n}, y\right\rangle\right) H(-i q ; \vec{u}) d \vec{u} \\
& =\phi_{0}\left(-i q ;\left\langle\alpha_{1}, y\right\rangle, \cdots,\left\langle\alpha_{n}, y\right\rangle\right)
\end{aligned}
$$

as desired.

Theorem 4.2. Let $q \in \mathbb{R}-\{0\}$ and $F \in \mathcal{B}(1 ; m)$ be as in Theorem 4.1. Furthermore, assume that for each $l \in\{1,2, \cdots, n\}$,

$$
\exp \left\{-\sum_{j=1}^{n} \frac{\sqrt{2|q|} A_{j} \xi_{j}}{2 B_{j}}\right\} \mathcal{F}\left(f_{l}(\cdot) H(-i q ; \cdot)\right)\left(-\frac{q \xi_{1}}{B_{1}}, \cdots,-\frac{q \xi_{n}}{B_{n}}\right)
$$

belongs to $C_{0}\left(\mathbb{R}^{n}\right)$. Then for each $l \in\{1,2, \cdots, n\}$,

$$
\phi_{l}(-i q ; \vec{\xi}) \equiv\left(\prod_{j=1}^{n} \frac{-i q}{2 \pi B_{j}}\right)^{\frac{1}{2}} \int_{\mathbb{R}^{n}} f_{l}(\vec{\xi}+\vec{u}) H(-i q ; \vec{u}) d \vec{u}
$$

is an element of $C_{0}\left(\mathbb{R}^{n}\right)$. In addition, for each $w \in A$ and s-a.e. $y \in C_{a, b}[0, T]$,

$$
\begin{aligned}
\delta T_{q}(1 ; F)(y \mid w) & =\sum_{l=1}^{n}\left\langle\alpha_{l}, w\right\rangle \phi_{l}\left(-i q ;\left\langle\alpha_{1}, y\right\rangle, \cdots,\left\langle\alpha_{n}, y\right\rangle\right) \\
& =T_{q}(1 ; \delta F(\cdot \mid w))(y),
\end{aligned}
$$

which, as a function of $y$, is an element of $\mathcal{B}(\infty ; m-1)$.

Proof. The proof that each $\phi_{l}(-i q ; \cdot)$ belongs to $C_{0}\left(\mathbb{R}^{n}\right)$ is the same as the proof in Theorem 4.1 above showing that $\phi_{0}(-i q ; \cdot) \in C_{0}\left(\mathbb{R}^{n}\right)$. Equation (4.12) then follows immediately using the definition of the first variation and equation (4.7).

Our next theorem gives a parts formula involving $F$ and $T_{q}(1 ; G)$.

Theorem 4.3. Let $q \in \mathbb{R}-\{0\}$ be given and let $F \in \mathcal{B}(1 ; m)$ be as in Theorem 4.1. Let $G \in \mathcal{B}(1 ; m)$ be given by equation (3.2) with

$$
\int_{\mathbb{R}^{n}}\left|g_{j_{1}, \cdots, j_{k}}(\vec{u})\right| \exp \left\{\left(\frac{1+|q|}{2}\right)^{\frac{1}{2}} \sum_{j=1}^{n} \frac{\left|A_{j} u_{j}\right|}{B_{j}}\right\} d \vec{u}<\infty
$$

for all $k \in\{0,1, \cdots, m\}$ and each $j_{i} \in\{1, \cdots, n\}$. Furthermore, assume that

$$
\exp \left\{-\sum_{j=1}^{n} \frac{\sqrt{2|q|} A_{j} \xi_{j}}{2 B_{j}}\right\} \mathcal{F}\left(g_{l}(\cdot) H(-i q ; \cdot)\right)\left(-\frac{q \xi_{1}}{B_{1}}, \cdots,-\frac{q \xi_{n}}{B_{n}}\right)
$$


belongs to $C_{0}\left(\mathbb{R}^{n}\right)$ for all $l \in\{0,1, \cdots, n\}$. Let $z \in L_{a, b}^{2}[0, T]$ be given and for $t \in[0, T]$, let $w(t)=\int_{0}^{t} z(s) d b(s)$. Then

$$
\begin{aligned}
E_{x}^{\operatorname{anf}_{q}}[F(x) & \left.\delta T_{q}(1 ; G)(x \mid w)+\delta F(x \mid w) T_{q}(1 ; G)(x)\right] \\
=- & i q E_{x}^{\operatorname{anf}_{q}}\left[F(x) T_{q}(1 ; G)(x)\langle z, x\rangle\right] \\
& -(-i q)^{\frac{1}{2}}\left(z, a^{\prime}\right) E_{x}^{\operatorname{anf}_{q}}\left[F(x) T_{q}(1 ; G)(x)\right] .
\end{aligned}
$$

Proof. Let $R(x)=F(x) T_{q}(1 ; G)(x)$. By Theorem $4.1, T_{q}(1 ; G)(x)$ is an element of $\mathcal{B}(\infty ; m)$ and so $R(x)$ is an element of $\mathcal{B}(1 ; m)$. Also, by Theorem 4.1 , Theorem 4.2 and Lemma 3.2 ,

$$
\delta R(x \mid w)=F(x) \delta T_{q}(1 ; G)(x \mid w)+\delta F(x \mid w) T_{q}(1 ; G)(x),
$$

as a function of $x$, is an element of $\mathcal{B}(1 ; m-1)$. In addition, we know that for each $l \in\{0,1, \cdots, n\}$,

$$
\psi_{l}(-i q ; \vec{v}) \equiv\left(\prod_{j=1}^{n} \frac{-i q}{2 \pi B_{j}}\right)^{1 / 2} \int_{\mathbb{R}^{n}} g_{l}(\vec{u}+\vec{v}) H(-i q ; \vec{u}) d \vec{u}
$$

is an element of $C_{0}\left(\mathbb{R}^{n}\right)$ with

$$
T_{q}(1 ; G)(y)=\psi_{0}\left(-i q ;\left\langle\alpha_{1}, y\right\rangle, \cdots,\left\langle\alpha_{n}, y\right\rangle\right)
$$

and

$$
\delta T_{q}(1 ; G)(y \mid w)=\sum_{l=1}^{n}\left\langle\alpha_{l}, w\right\rangle \psi_{l}\left(-i q ;\left\langle\alpha_{1}, y\right\rangle, \cdots,\left\langle\alpha_{n}, y\right\rangle\right)
$$

for s-a.e. $y \in C_{a, b}[0, T]$. Hence, both of the following analytic Feynman integrals exist:

$$
\begin{aligned}
E_{x}^{\operatorname{anf}_{q}}[R(x)]=E_{x}^{\operatorname{anf}_{q}}\left[F(x) T_{q}(1 ; G)(x)\right] \\
=\left(\prod_{j=1}^{n} \frac{-i q}{2 \pi B_{j}}\right)^{1 / 2} \int_{\mathbb{R}^{n}} f(\vec{u}) \psi_{0}(-i q ; \vec{u}) H(-i q ; \vec{u}) d \vec{u}
\end{aligned}
$$

and

$$
\begin{aligned}
& E_{x}^{\operatorname{anf}_{q}[}[\delta R(x \mid w)]=E_{x}^{\operatorname{anf}_{q}}[F(x)\left.\delta T_{q}(1 ; G)(x \mid w)+\delta F(x \mid w) T_{q}(1 ; G)(x)\right] \\
&=\left(\prod_{j=1}^{n} \frac{-i q}{2 \pi B_{j}}\right)^{1 / 2} \int_{\mathbb{R}^{n}}\left[f(\vec{u}) \sum_{l=1}^{n}\left\langle\alpha_{l}, w\right\rangle \psi_{l}(-i q ; \vec{u})\right. \\
&\left.+\psi_{0}(-i q ; \vec{u}) \sum_{l=1}^{n}\left\langle\alpha_{l}, w\right\rangle f_{l}(\vec{u})\right] H(-i q ; \vec{u}) d \vec{u} .
\end{aligned}
$$

Also, proceeding as in the proof of Theorem 3.3 above, it is easy to show that for $\rho>0$ and $h>0$,

$$
\begin{aligned}
& E[|\delta R(\rho x+\rho h w \mid \rho w)|] \\
& \leq \rho\|z\|_{a, b}\left(\prod_{j=1}^{n} 2 \pi \rho^{2} B_{j}\right)^{-1 / 2}\left[\|f(\cdot)\|_{1} \sum_{l=1}^{n}\left\|\psi_{l}(-i q ; \cdot)\right\|_{\infty}\right. \\
& \left.+\left\|\psi_{0}(-i q ; \cdot)\right\|_{\infty} \sum_{l=1}^{n}\left\|f_{l}(\cdot)\right\|_{1}\right]<\infty .
\end{aligned}
$$


Hence, by Theorem 2.1 above, the analytic Feynman integral

$$
E_{x}^{\operatorname{anf}_{q}}[R(x)\langle z, x\rangle]=E_{x}^{\operatorname{anf}_{q}}\left[F(x) T_{q}(1 ; G)(x)\langle z, x\rangle\right]
$$

exists and equality (4.14) holds.

Choosing $G=F$ in Theorem 4.3 we get the following integration by parts formula.

Corollary 4.4. Let $q \in \mathbb{R}-\{0\}$ be given and let $F \in \mathcal{B}(1 ; m)$ be as in Theorem 4.2. Let $z$ and $w$ be as in Theorem 4.3. Then

$$
\begin{aligned}
& E_{x}^{\operatorname{anf}_{q}}\left[F(x) \delta T_{q}(1 ; F)(x \mid w)+\delta F(x \mid w) T_{q}(1 ; F)(x)\right] \\
& =-i q E_{x}^{\operatorname{anf}_{q}}\left[F(x) T_{q}(1 ; F)(x)\langle z, x\rangle\right] \\
& -(-i q)^{\frac{1}{2}}\left(z, a^{\prime}\right) E_{x}^{\operatorname{anf}_{q}}\left[F(x) T_{q}(1 ; F)(x)\right] .
\end{aligned}
$$

Next we obtain a parts formula involving $T_{q}(1 ; F)$ and $T_{q}(1 ; G)$.

Theorem 4.5. Let $q \in \mathbb{R}-\{0\}$. Let $F \in \mathcal{B}(1 ; m)$ be as in Theorem 4.2 and let $G \in$ $\mathcal{B}(1 ; m)$ be as in Theorem 4.3. Furthermore, assume that for each $l \in\{0,1, \cdots, n\}$,

$$
\int_{\mathbb{R}^{n}}\left|\psi_{l}(-i q ; \vec{u}) H(-i q ; \vec{u})\right| d \vec{u}<\infty .
$$

Then for $w(t)=\int_{0}^{t} z(s) d b(s)$ with $z \in L_{a, b}^{2}[0, T]$,

$$
\begin{aligned}
E_{x}^{\operatorname{anf}_{q}} & {\left[T_{q}(1 ; F)(x) \delta T_{q}(1 ; G)(x \mid w)+\delta T_{q}(1 ; F)(x \mid w) T_{q}(1 ; G)(x)\right] } \\
=- & i q E_{x}^{\operatorname{anf}_{q}}\left[T_{q}(1 ; F)(x) T_{q}(1 ; G)(x)\langle z, x\rangle\right] \\
& -(-i q)^{\frac{1}{2}}\left(z, a^{\prime}\right) E_{x}^{\operatorname{anf}_{q}}\left[T_{q}(1 ; F)(x) T_{q}(1 ; G)(x)\right] .
\end{aligned}
$$

Proof. Let $R(x)=T_{q}(1 ; F)(x) T_{q}(1 ; G)(x)$. Then $R \in \mathcal{B}(\infty ; m)$ and $\delta R(x \mid w)$, as a function of $x$, is an element of $\mathcal{B}(\infty ; m-1)$. Hence, by (4.6), (4.11) and (4.20), both of the following analytic Feynman integrals exist:

$$
E_{x}^{\operatorname{anf}_{q}}[R(x)]=\left(\prod_{j=1}^{n} \frac{-i q}{2 \pi B_{j}}\right)^{\frac{1}{2}} \int_{\mathbb{R}^{n}} \phi_{0}(-i q ; \vec{u}) \psi_{0}(-i q ; \vec{u}) H(-i q ; \vec{u}) d \vec{u}
$$

and

$$
\begin{aligned}
& E_{x}^{\operatorname{anf}_{q}}[\delta R(x \mid w)] \\
& =\left(\prod_{j=1}^{n} \frac{-i q}{2 \pi B_{j}}\right)^{\frac{1}{2}} \int_{\mathbb{R}^{n}}\left[\phi_{0}(-i q ; \vec{u}) \sum_{l=1}^{n}\left\langle\alpha_{l}, w\right\rangle \psi_{l}(-i q ; \vec{u})\right. \\
& \left.+\psi_{0}(-i q ; \vec{u}) \sum_{l=1}^{n}\left\langle\alpha_{l}, w\right\rangle \phi_{l}(-i q ; \vec{u})\right] H(-i q ; \vec{u}) d \vec{u} .
\end{aligned}
$$

In addition, for $\rho>0$ and $h>0$,

$$
\begin{aligned}
& E[|\delta R(\rho x+\rho h w \mid \rho w)|] \\
& \leq \rho\|z\|_{a, b}\left[\left\|\phi_{0}(-i q ; \cdot)\right\|_{\infty} \sum_{l=1}^{n}\left\|\psi_{l}(-i q ; \cdot)\right\|_{\infty}\right. \\
& \left.+\left\|\psi_{0}(-i q ; \cdot)\right\|_{\infty} \sum_{l=1}^{n}\left\|\phi_{l}(-i q ; \cdot)\right\|_{\infty}\right]<\infty .
\end{aligned}
$$


Hence, by Theorem 2.1, the analytic Feynman integral $E_{x}^{\mathrm{anf}_{q}}[R(x)\langle z, x\rangle]$ exists and equality (4.21) holds.

We finish this section with some examples which shed light upon the necessity of conditions such as (4.4) and (4.5), and which also illustrate that the conclusions of Lemma 3.7 are not necessarily valid for $\lambda \in \tilde{\mathbb{C}}_{+}$with $\operatorname{Re}(\lambda)=0$.

In our first example we define a functional $F$ of the form (1.1) with $n=1$, such that $F$ is an element of $\mathcal{B}(p ; m)$ for all $p \in[1,+\infty], f$ is an element of $L^{p}(\mathbb{R})$ for all $p \in[1,+\infty]$, and yet $\phi_{0}(i ; \cdot)$ given by $(4.6)$ is not an element of $C_{0}(\mathbb{R})$. In fact, $\left|\phi_{0}\left(i ; \xi_{1}\right)\right|=+\infty$ for all $\xi_{1} \in \mathbb{R}$.

Example 4.6. Let $q=-1$, let $n=1$, let $m$ be a nonnegative integer, and let $\alpha_{1}$ be an element of $L_{a, b}^{2}[0, T]$ with $\left\|\alpha_{1}\right\|_{a, b}=1$. Without loss of generality, we will assume that $A_{1}$ (see equation (2.18)) is positive.

Let $f: \mathbb{R} \rightarrow \mathbb{C}$ be defined by the formula

$$
f\left(u_{1}\right) \equiv u_{1}^{m+1} \chi_{[0,+\infty)}\left(u_{1}\right) \exp \left\{\frac{i u_{1}^{2}}{2 B_{1}}-\frac{i \sqrt{2} A_{1} u_{1}}{2 B_{1}}+\frac{A_{1}^{2}}{2 B_{1}}-\frac{\sqrt{2} A_{1} u_{1}}{4 B_{1}}\right\} .
$$

We note that

$$
\left|f\left(u_{1}\right)\right|=u_{1}^{m+1} \chi_{[0,+\infty)}\left(u_{1}\right) \exp \left\{\frac{A_{1}^{2}}{2 B_{1}}-\frac{\sqrt{2} A_{1} u_{1}}{4 B_{1}}\right\},
$$

and hence $f \in L^{p}(\mathbb{R})$ for all $p \in[1,+\infty]$. In fact, $f$ is also an element of $C_{0}(\mathbb{R})$. We then define $F: C_{a, b}[0, T] \rightarrow \mathbb{C}$ by the formula

$$
F(x) \equiv f\left(\left\langle\alpha_{1}, x\right\rangle\right)
$$

It is easy to see that $F \in \mathcal{B}(p ; m)$ for all $p \in[1,+\infty]$.

Next, using equation (3.4) with $n=1, \lambda=i$, and $\sqrt{i}=\frac{1+i}{\sqrt{2}}$, we observe that

$$
H\left(i ; u_{1}\right)=\exp \left\{\frac{\sqrt{2} A_{1} u_{1}+i \sqrt{2} A_{1} u_{1}-A_{1}^{2}-i u_{1}^{2}}{2 B_{1}}\right\},
$$

and hence

$$
f\left(u_{1}\right) H\left(i ; u_{1}\right)=u_{1}^{m+1} \chi_{[0,+\infty)}\left(u_{1}\right) \exp \left\{\frac{\sqrt{2} A_{1} u_{1}}{4 B_{1}}\right\},
$$

which is not an element of $L^{p}(\mathbb{R})$ for any $p \in[1,+\infty]$.

Then, using equation (4.6) with $n=1$ and $q=-1$, equation (4.25) and equation (4.28), we see that

$$
\begin{aligned}
\phi_{0}\left(i ; \xi_{1}\right)= & \left(\frac{i}{2 \pi B_{1}}\right)^{\frac{1}{2}} \int_{\mathbb{R}} f\left(u_{1}+\xi_{1}\right) H\left(i ; u_{1}\right) d u_{1} \\
= & \left(\frac{i}{2 \pi B_{1}}\right)^{\frac{1}{2}} \exp \left\{\frac{i \xi_{1}^{2}}{2 B_{1}}-\frac{i \sqrt{2} A_{1} \xi_{1}}{2 B_{1}}-\frac{\sqrt{2} A_{1} \xi_{1}}{4 B_{1}}\right\} \\
& \cdot \int_{\mathbb{R}}\left(u_{1}+\xi_{1}\right)^{m+1} \chi_{[0,+\infty)}\left(u_{1}+\xi_{1}\right) \exp \left\{\frac{i u_{1} \xi_{1}}{B_{1}}+\frac{\sqrt{2} A_{1} u_{1}}{4 B_{1}}\right\} d u_{1} \\
= & \left(\frac{i}{2 \pi B_{1}}\right)^{\frac{1}{2}} \exp \left\{\frac{i \xi_{1}^{2}}{2 B_{1}}-\frac{i \sqrt{2} A_{1} \xi_{1}}{2 B_{1}}-\frac{\sqrt{2} A_{1} \xi_{1}}{4 B_{1}}\right\} \\
& \cdot \int_{-\xi_{1}}^{+\infty}\left(u_{1}+\xi_{1}\right)^{m+1} \exp \left\{\frac{i u_{1} \xi_{1}}{B_{1}}+\frac{\sqrt{2} A_{1} u_{1}}{4 B_{1}}\right\} d u_{1} .
\end{aligned}
$$


Thus,

$$
\begin{aligned}
\left|\phi_{0}\left(i ; \xi_{1}\right)\right|=\left(2 \pi B_{1}\right)^{-\frac{1}{2}} \exp \left\{-\frac{\sqrt{2} A_{1} \xi_{1}}{4 B_{1}}\right\} \\
\cdot\left|\int_{-\xi_{1}}^{+\infty}\left(u_{1}+\xi_{1}\right)^{m+1} \exp \left\{\frac{i u_{1} \xi_{1}}{B_{1}}+\frac{\sqrt{2} A_{1} u_{1}}{4 B_{1}}\right\} d u_{1}\right| .
\end{aligned}
$$

Hence, choosing $\xi_{1}=0$, and using the fact that $A_{1}$ is positive, we see that

$$
\begin{aligned}
\left|\phi_{0}(i ; 0)\right| & =\left(2 \pi B_{1}\right)^{-\frac{1}{2}}\left|\int_{0}^{+\infty} u^{m+1} \exp \left\{\frac{\sqrt{2} A_{1} u_{1}}{4 B_{1}}\right\} d u_{1}\right| \\
& =\left(2 \pi B_{1}\right)^{-\frac{1}{2}} \int_{0}^{+\infty} u^{m+1} \exp \left\{\frac{\sqrt{2} A_{1} u_{1}}{4 B_{1}}\right\} d u_{1}=+\infty,
\end{aligned}
$$

which implies that $\phi_{0}(i ; \cdot)$ is not an element of $C_{0}(\mathbb{R})$. In fact, for each fixed $\xi_{1} \in \mathbb{R}$, we observe that

$$
\begin{aligned}
&\left|\phi_{0}\left(i ; \xi_{1}\right)\right|=\left(2 \pi B_{1}\right)^{-\frac{1}{2}} \exp \left\{-\frac{\sqrt{2} A_{1} \xi_{1}}{4 B_{1}}\right\} \\
& \cdot\left|\int_{-\xi_{1}}^{+\infty}\left(u_{1}+\xi_{1}\right)^{m+1} \exp \left\{\frac{i u_{1} \xi_{1}}{B_{1}}+\frac{\sqrt{2} A_{1} u_{1}}{4 B_{1}}\right\} d u_{1}\right|=+\infty,
\end{aligned}
$$

and so $\phi_{0}(i ; \cdot)$ is not an element of $L^{p}(\mathbb{R})$ for any $p \in[1,+\infty]$ even though $f(\cdot)$ was an element of $L^{p}(\mathbb{R})$ for all $p \in[1,+\infty]$ and $F$ was an element of $\mathcal{B}(p ; m)$ for all $p \in[1,+\infty]$. Hence, the $L_{1}$ analytic GFFT, $T_{-1}(1 ; F)$ does not exist.

We also note that $f$ does not satisfy condition (4.4) above since by equation (4.26) (recall that $q=-1$ and so $\left(\frac{1+|q|}{2}\right)^{1 / 2}=1$ ),

$$
\begin{aligned}
\int_{\mathbb{R}}\left|f\left(u_{1}\right)\right| \exp \left\{\frac{\left|A_{1} u_{1}\right|}{B_{1}}\right\} d u_{1} \\
\quad=\int_{0}^{\infty} u_{1}^{m+1} \exp \left\{\frac{A_{1}^{2}}{2 B_{1}}-\frac{\sqrt{2} A_{1} u_{1}}{4 B_{1}}+\frac{A_{1} u_{1}}{B_{1}}\right\} d u_{1}=+\infty .
\end{aligned}
$$

In our next example we exhibit a functional $F$ of the form (1.1) that satisfies conditions (4.4) and (4.5) above. Furthermore, we are able to evaluate the integral in equation (4.6) and thus obtain a formula for $\phi_{0}(i ; \vec{\xi})$ which does not involve any integrals.

Example 4.7. Let $q=-1$, let $m$ be a nonnegative integer and let $n$ be a positive integer. Let $\left\{\alpha_{1}, \cdots, \alpha_{n}\right\}$ be an orthonormal set of functions from $\left(L_{a, b}^{2}[0, T]\right.$, $\left.\|\cdot\|_{a, b}\right)$, and for each $j \in\{1, \cdots, n\}$ let $A_{j}$ and $B_{j}$ be given by (2.18) and (2.19) respectively. We define $f: \mathbb{R}^{n} \rightarrow \mathbb{C}$ by the formula

$$
f(\vec{u}) \equiv \exp \left\{\sum_{j=1}^{n} \frac{\left[i u_{j}^{2}-i \sqrt{2} A_{j} u_{j}+A_{j}^{2}-u_{j}^{2}-\sqrt{2} A_{j} u_{j}\right]}{2 B_{j}}\right\} .
$$

We note that

$$
|f(\vec{u})|=\exp \left\{\sum_{j=1}^{n} \frac{\left[A_{j}^{2}-u_{j}^{2}-\sqrt{2} A_{j} u_{j}\right]}{2 B_{j}}\right\},
$$

and hence $f \in L^{p}\left(\mathbb{R}^{n}\right)$ for all $p \in[1,+\infty]$. Also, $f \in C_{0}\left(\mathbb{R}^{n}\right)$. 
Let $F: C_{a, b}[0, T] \rightarrow \mathbb{C}$ be given by

$$
F(x) \equiv f\left(\left\langle\alpha_{1}, x\right\rangle, \cdots,\left\langle\alpha_{n}, x\right\rangle\right) .
$$

It is easy to show that $F \in \mathcal{B}(p ; m)$ for all $p \in[1,+\infty]$.

Next, using equation (4.33), together with equation (3.4) with $\lambda=i$ and $\sqrt{i}=$ $\frac{1+i}{\sqrt{2}}$, it follows that

$$
f(\vec{u}) H(i ; \vec{u})=\exp \left\{-\sum_{j=1}^{n} \frac{u_{j}^{2}}{2 B_{j}}\right\} .
$$

Now clearly $f(\cdot) H(i ; \cdot)$ is an element of $L^{p}\left(\mathbb{R}^{n}\right) \cap C_{0}\left(\mathbb{R}^{n}\right)$ for all $p \in[1,+\infty]$. Next, using equations (4.6), (3.4) and (4.33) we obtain

$$
\begin{aligned}
\phi_{0}(i ; \vec{\xi})= & \left(\prod_{j=1}^{n} \frac{i}{2 \pi B_{j}}\right)^{\frac{1}{2}} \int_{\mathbb{R}^{n}} f(\vec{u}+\vec{\xi}) H(i ; \vec{u}) d \vec{u} \\
= & \left(\prod_{j=1}^{n} \frac{i}{2 \pi B_{j}}\right)^{\frac{1}{2}} \exp \left\{\sum_{j=1}^{n} \frac{\left[i \xi_{j}^{2}-i \sqrt{2} A_{j} \xi_{j}-\sqrt{2} A_{j} \xi_{j}\right]}{2 B_{j}}\right\} \\
& \cdot \int_{\mathbb{R}^{n}} \exp \left\{i \sum_{j=1}^{n} \frac{u_{j} \xi_{j}}{B_{j}}-\sum_{j=1}^{n} \frac{\left(u_{j}+\xi_{j}\right)^{2}}{2 B_{j}}\right\} d \vec{u} \\
= & \left(\prod_{j=1}^{n} \frac{i}{2 \pi B_{j}}\right)^{\frac{1}{2}} \exp \left\{-\sum_{j=1}^{n} \frac{\left[i \xi_{j}^{2}+i \sqrt{2} A_{j} \xi_{j}+\sqrt{2} A_{j} \xi_{j}\right]}{2 B_{j}}\right\} \\
& \cdot \int_{\mathbb{R}^{n}} \exp \left\{i \sum_{j=1}^{n} \frac{u_{j} \xi_{j}}{B_{j}}-\sum_{j=1}^{n} \frac{u_{j}^{2}}{2 B_{j}}\right\} d \vec{u} \\
= & (i)^{\frac{n}{2}} \exp \left\{-\sum_{j=1}^{n} \frac{\left[i \xi_{j}^{2}+i \sqrt{2} A_{j} \xi_{j}+\sqrt{2} A_{j} \xi_{j}+\xi_{j}^{2}\right]}{2 B_{j}}\right\},
\end{aligned}
$$

because

$$
\begin{aligned}
\int_{\mathbb{R}^{n}} & \exp \left\{i \sum_{j=1}^{n} \frac{u_{j} \xi_{j}}{B_{j}}-\sum_{j=1}^{n} \frac{u_{j}^{2}}{2 B_{j}}\right\} d \vec{u} \\
& =\left(\prod_{j=1}^{n} 2 \pi B_{j}\right)^{\frac{1}{2}} \exp \left\{-\sum_{j=1}^{n} \frac{\xi_{j}^{2}}{2 B_{j}}\right\} \\
& =(2 \pi)^{\frac{n}{2}} \mathcal{F}(f(\cdot) H(i ; \cdot))\left(\frac{\xi_{1}}{B_{1}}, \cdots, \frac{\xi_{n}}{B_{n}}\right) .
\end{aligned}
$$

Hence,

$$
\left|\phi_{0}(i ; \vec{\xi})\right|=\exp \left\{-\sum_{j=1}^{n} \frac{\left[\sqrt{2} A_{j} \xi_{j}+\xi_{j}^{2}\right]}{2 B_{j}}\right\},
$$

and so $\phi_{0}(i ; \cdot)$ is an element of $C_{0}\left(\mathbb{R}^{n}\right) \cap L^{p}\left(\mathbb{R}^{n}\right)$ for all $p \in[1,+\infty]$.

We also note that because of the factor $\exp \left\{-\frac{u_{j}^{2}}{2 B_{j}}\right\}$ in the definition of $f(\vec{u})$ given by equation (4.33), condition (4.4) certainly holds. In addition, condition 
(4.5) holds because (recall $q=-1$ )

$$
\begin{aligned}
\exp \{ & \left.-\sum_{j=1}^{n} \frac{\sqrt{2} A_{j} \xi_{j}}{2 B_{j}}\right\} \mathcal{F}(f(\cdot) H(i ; \cdot))\left(\frac{\xi_{1}}{B_{1}}, \cdots, \frac{\xi_{n}}{B_{n}}\right) \\
= & \exp \left\{-\sum_{j=1}^{n} \frac{\sqrt{2} A_{j} \xi_{j}}{2 B_{j}}\right\}\left(\prod_{j=1}^{n} B_{j}\right)^{\frac{1}{2}} \exp \left\{-\sum_{j=1}^{n} \frac{\xi_{j}^{2}}{2 B_{j}}\right\} \\
= & \left(\prod_{j=1}^{n} B_{j}\right)^{\frac{1}{2}} \exp \left\{-\sum_{j=1}^{n} \frac{\left[\sqrt{2} A_{j} \xi_{j}+\xi_{j}^{2}\right]}{2 B_{j}}\right\} \\
= & \left(\prod_{j=1}^{n} B_{j}\right)^{\frac{1}{2}}\left|\phi_{0}(i ; \vec{\xi})\right|
\end{aligned}
$$

is an element of $C_{0}\left(\mathbb{R}^{n}\right)$ as was shown above. Hence, by Theorem 4.1 , the $L_{1}$ analytic GFFT, $T_{-1}(1 ; F)$ exists as an element of $\mathcal{B}(\infty ; m)$ and for s-a.e. $y \in$ $C_{a, b}[0, T]$ is given by the formula

$$
\begin{aligned}
& T_{-1}(1 ; F)(y) \\
& =\phi_{0}\left(i ;\left\langle\alpha_{1}, y\right\rangle, \cdots,\left\langle\alpha_{n}, y\right\rangle\right) \\
& =(i)^{\frac{n}{2}} \exp \left\{-\sum_{j=1}^{n} \frac{\left[(1+i)\left\langle\alpha_{j}, y\right\rangle^{2}+(1+i) \sqrt{2} A_{j}\left\langle\alpha_{j}, y\right\rangle\right]}{2 B_{j}}\right\} .
\end{aligned}
$$

5. Parts Formulas involving $T_{q}(2 ; F)$ And $T_{q}(2 ; G)$

Note that in our first theorem below we replace conditions (4.4) and (4.5) with condition (5.1). This condition is used to obtain a dominating function in order to apply the dominated convergence theorem.

Theorem 5.1. Let $q \in \mathbb{R}-\{0\}$ be given. Let $F \in \mathcal{B}(2 ; m)$ be given by equation (1.1) with

$$
\int_{\mathbb{R}^{n}}\left[\int_{\mathbb{R}^{n}}\left|f_{j_{1}, \cdots, j_{k}}(\vec{\xi}+\vec{u})\right| \exp \left\{\left(\frac{1+|q|}{2}\right)^{\frac{1}{2}} \sum_{j=1}^{n} \frac{\left|A_{j} u_{j}\right|}{B_{j}}\right\} d \vec{u}\right]^{2} d \vec{\xi}<\infty
$$

for all $k \in\{0,1, \cdots, m\}$ and each $j_{i} \in\{1, \cdots, n\}$. Then

$$
\phi_{0}(-i q ; \vec{\xi}) \equiv\left(\prod_{j=1}^{n} \frac{-i q}{2 \pi B_{j}}\right)^{\frac{1}{2}} \int_{\mathbb{R}^{n}} f(\vec{\xi}+\vec{u}) H(-i q ; \vec{u}) d \vec{u}
$$

is an element of $L^{2}\left(\mathbb{R}^{n}\right)$. Furthermore, the $L_{2}$ analytic $G F F T, T_{q}(2 ; F)$ exists as an element of $\mathcal{B}(2 ; m)$ and for s-a.e. $y \in C_{a, b}[0, T]$ is given by the formula

$$
T_{q}(2 ; F)(y)=\phi_{0}\left(-i q ;\left\langle\alpha_{1}, y\right\rangle, \cdots,\left\langle\alpha_{n}, y\right\rangle\right) \text {. }
$$

Proof. Using (4.1) we first note that

$$
\begin{aligned}
\left|\phi_{0}(-i q ; \vec{\xi})\right| & \leq\left(\prod_{j=1}^{n} \frac{|q|}{2 \pi B_{j}}\right)^{\frac{1}{2}} \int_{\mathbb{R}^{n}}|f(\vec{\xi}+\vec{u}) H(-i q ; \vec{u})| d \vec{u} \\
& \leq\left(\prod_{j=1}^{n} \frac{|q|}{2 \pi B_{j}}\right)^{\frac{1}{2}} \int_{\mathbb{R}^{n}}|f(\vec{\xi}+\vec{u})| \exp \left\{\left(\frac{1+|q|}{2}\right)^{\frac{1}{2}} \sum_{j=1}^{n} \frac{\left|A_{j} u_{j}\right|}{B_{j}}\right\} d \vec{u} .
\end{aligned}
$$


Hence, by (5.1) with $k=0$,

$$
\begin{aligned}
& \left\|\phi_{0}(-i q ; \cdot)\right\|_{2}^{2}=\int_{\mathbb{R}^{n}}\left|\phi_{0}(-i q ; \vec{\xi})\right|^{2} d \vec{\xi} \\
& \leq\left(\prod_{j=1}^{n} \frac{|q|}{2 \pi B_{j}}\right) \int_{\mathbb{R}^{n}}\left[\int_{\mathbb{R}^{n}}|f(\vec{\xi}+\vec{u})| \exp \left\{\left(\frac{1+|q|}{2}\right)^{\frac{1}{2}} \sum_{j=1}^{n} \frac{\left|A_{j} u_{j}\right|}{B_{j}}\right\} d \vec{u}\right]^{2} d \vec{\xi}<\infty
\end{aligned}
$$

and so $\phi_{0}(-i q ; \vec{\xi})$ is an element of $L^{2}\left(\mathbb{R}^{n}\right)$.

To show that $T_{q}(2 ; F)$ exists and is given by equation (5.3) it suffices to show that for each $\rho>0$,

$$
\lim _{\lambda \rightarrow-i q} \int_{C_{a, b}[0, T]}\left|T_{\lambda}(\rho y)-\phi_{0}\left(-i q ;\left\langle\alpha_{1}, \rho y\right\rangle, \cdots,\left\langle\alpha_{n}, \rho y\right\rangle\right)\right|^{2} d \mu(y)=0 .
$$

But

$$
\begin{aligned}
& \int_{C_{a, b}[0, T]}\left|T_{\lambda}(\rho y)-\phi_{0}\left(-i q ;\left\langle\alpha_{1}, \rho y\right\rangle, \cdots,\left\langle\alpha_{n}, \rho y\right\rangle\right)\right|^{2} d \mu(y) \\
& =\int_{C_{a, b}[0, T]} \mid \phi_{0}\left(\lambda ;\left\langle\alpha_{1}, \rho y\right\rangle, \cdots,\left\langle\alpha_{n}, \rho y\right\rangle\right) \\
& \quad-\left.\phi_{0}\left(-i q ;\left\langle\alpha_{1}, \rho y\right\rangle, \cdots,\left\langle\alpha_{n}, \rho y\right\rangle\right)\right|^{2} d \mu(y) \\
& =\left(\prod_{j=1}^{n} 2 \pi B_{j} \rho^{2}\right)^{-\frac{1}{2}} \int_{\mathbb{R}^{n}}\left|\phi_{0}(\lambda ; \vec{u})-\phi_{0}(-i q ; \vec{u})\right|^{2} \exp \left\{-\sum_{j=1}^{n} \frac{\left(u_{j}-\rho A_{j}\right)^{2}}{2 \rho^{2} B_{j}}\right\} d \vec{u} \\
& \leq\left(\prod_{j=1}^{n} 2 \pi B_{j} \rho^{2}\right)^{-\frac{1}{2}}\left\|\phi_{0}(\lambda ; \cdot)-\phi_{0}(-i q ; \cdot)\right\|_{2}^{2} .
\end{aligned}
$$

Now clearly $\phi_{0}(\lambda ; \vec{\xi}) \rightarrow \phi_{0}(-i q ; \vec{\xi})$ a.e. on $\mathbb{R}^{n}$ as $\lambda \rightarrow-i q$ through values in $\mathbb{C}_{+}$. Thus, to show that

$$
\left\|\phi_{0}(\lambda ; \cdot)-\phi_{0}(-i q ; \cdot)\right\|_{2} \rightarrow 0
$$

it suffices [11, p. 126] to show that

$$
\left\|\phi_{0}(\lambda ; \cdot)\right\|_{2} \rightarrow\left\|\phi_{0}(-i q ; \cdot)\right\|_{2}
$$

as $\lambda \rightarrow-i q$ through values in $\mathbb{C}_{+}$. But for all $\lambda \in \mathbb{C}_{+}$with $\operatorname{Re}(\sqrt{\lambda})<((1+|q|) / 2)^{\frac{1}{2}}$,

$$
\begin{aligned}
& \int_{\mathbb{R}^{n}}\left|\int_{\mathbb{R}^{n}} f(\vec{\xi}+\vec{u}) H(\lambda ; \vec{u}) d \vec{u}\right|^{2} d \vec{\xi} \\
& \leq \int_{\mathbb{R}^{n}}\left[\int_{\mathbb{R}^{n}}|f(\vec{\xi}+\vec{u})| \exp \left\{\left(\frac{1+|q|}{2}\right)^{\frac{1}{2}} \sum_{j=1}^{n} \frac{\left|A_{j} u_{j}\right|}{B_{j}}\right\} d \vec{u}\right]^{2} d \vec{\xi}<\infty .
\end{aligned}
$$


Hence, by the dominated convergence theorem,

$$
\begin{aligned}
& \lim _{\lambda \rightarrow-i q}\left\|\phi_{0}(\lambda ; \cdot)\right\|_{2}^{2} \\
& =\lim _{\lambda \rightarrow-i q} \int_{\mathbb{R}^{n}}\left|\left(\prod_{j=1}^{n} \frac{\lambda}{2 \pi B_{j}}\right)^{\frac{1}{2}} \int_{\mathbb{R}^{n}} f(\vec{u}+\vec{\xi}) H(\lambda ; \vec{u}) d \vec{u}\right|^{2} d \vec{\xi} \\
& =\lim _{\lambda \rightarrow-i q}\left(\prod_{j=1}^{n} \frac{|\lambda|}{2 \pi B_{j}}\right) \int_{\mathbb{R}^{n}}\left|\int_{\mathbb{R}^{n}} f(\vec{u}+\vec{\xi}) H(\lambda ; \vec{u}) d \vec{u}\right|^{2} d \vec{\xi} \\
& =\left(\prod_{j=1}^{n} \frac{|q|}{2 \pi B_{j}}\right) \int_{\mathbb{R}^{n}}\left|\int_{\mathbb{R}^{n}} f(\vec{u}+\vec{\xi}) H(-i q ; \vec{u}) d \vec{u}\right|^{2} d \vec{\xi} \\
& =\left\|\phi_{0}(-i q ; \cdot)\right\|_{2}^{2} .
\end{aligned}
$$

Corollary 5.2. Let $q \in \mathbb{R}-\{0\}$ and $F \in \mathcal{B}(2 ; m)$ be as in Theorem 5.1. Then for each $l \in\{1,2, \cdots, n\}$,

$$
\phi_{l}(-i q ; \vec{\xi}) \equiv\left(\prod_{j=1}^{n} \frac{-i q}{2 \pi B_{j}}\right)^{\frac{1}{2}} \int_{\mathbb{R}^{n}} f_{l}(\vec{\xi}+\vec{u}) H(-i q ; \vec{u}) d \vec{u}
$$

is an element of $L^{2}\left(\mathbb{R}^{n}\right)$. In addition, for each $w \in A$ and s-a.e. $y \in C_{a, b}[0, T]$,

$$
\begin{aligned}
\delta T_{q}(2 ; F)(y \mid w) & =\sum_{l=1}^{n}\left\langle\alpha_{l}, w\right\rangle \phi_{l}\left(-i q ;\left\langle\alpha_{1}, y\right\rangle, \cdots,\left\langle\alpha_{n}, y\right\rangle\right) \\
& =T_{q}(2 ; \delta F(\cdot \mid w))(y),
\end{aligned}
$$

which, as a function of $y$, is an element of $\mathcal{B}(2 ; m-1)$.

Proof. The proof that each $\phi_{l}(-i q ; \cdot)$ belongs to $L^{2}\left(\mathbb{R}^{n}\right)$ is the same as the proof in Theorem 5.1 above showing that $\phi_{0}(-i q, \cdot) \in L^{2}\left(\mathbb{R}^{n}\right)$. Equation (5.5) then follows immediately using the definition of the first variation and equations (5.2) and (5.3).

Theorem 5.3. Let $q \in \mathbb{R}-\{0\}$ be given and let $F \in \mathcal{B}(2 ; m)$ be as in Theorem 5.1. Furthermore, assume that

$$
\int_{\mathbb{R}^{n}}\left|f_{j_{1}, \cdots, j_{k}}(\vec{u}) H(-i q ; \vec{u})\right|^{2} d \vec{u}<\infty
$$

for all $k \in\{0,1, \cdots, m\}$ and each $j_{i} \in\{1,2, \cdots, n\}$.

Let $G \in \mathcal{B}(2 ; m)$ be given by equation (3.2) with

$$
\int_{\mathbb{R}^{n}}\left[\int_{\mathbb{R}^{n}}\left|g_{j_{1}, \cdots, j_{k}}(\vec{\xi}+\vec{u})\right| \exp \left\{\left(\frac{1+|q|}{2}\right)^{\frac{1}{2}} \sum_{j=1}^{n} \frac{\left|A_{j} u_{j}\right|}{B_{j}}\right\} d \vec{u}\right]^{2} d \vec{\xi}<\infty
$$

for all $k \in\{0,1, \cdots, m\}$ and each $j_{i} \in\{1, \cdots, n\}$. Let $z$ and $w$ be as in Theorem 4.3. Then

$$
\begin{aligned}
E_{x}^{\operatorname{anf}_{q}}[F(x) & \left.\delta T_{q}(2 ; G)(x \mid w)+\delta F(x \mid w) T_{q}(2 ; G)(x)\right] \\
=- & i q E_{x}^{\operatorname{anf}_{q}}\left[F(x) T_{q}(2 ; G)(x)\langle z, x\rangle\right] \\
& -(-i q)^{\frac{1}{2}}\left(z, a^{\prime}\right) E_{x}^{\operatorname{anf}_{q}}\left[F(x) T_{q}(2 ; G)(x)\right] .
\end{aligned}
$$


Proof. By Theorem 5.1, for each $l \in\{0,1, \cdots, n\}$,

$$
\psi_{l}(-i q ; \vec{\xi}) \equiv\left(\prod_{j=1}^{n} \frac{-i q}{2 \pi B_{j}}\right)^{\frac{1}{2}} \int_{\mathbb{R}^{n}} g_{l}(\vec{\xi}+\vec{u}) H(-i q ; \vec{u}) d \vec{u}
$$

is an element of $L^{2}\left(\mathbb{R}^{n}\right)$. Furthermore,

$$
T_{q}(2 ; G)(x)=\psi_{0}\left(-i q ;\left\langle\alpha_{1}, x\right\rangle, \cdots,\left\langle\alpha_{n}, x\right\rangle\right)
$$

is an element of $\mathcal{B}(2 ; m)$, and as a function of $x$,

$$
\delta T_{q}(2 ; G)(x \mid w)=\sum_{l=1}^{n}\left\langle\alpha_{l}, w\right\rangle \psi_{l}\left(-i q ;\left\langle\alpha_{1}, x\right\rangle, \cdots,\left\langle\alpha_{n}, x\right\rangle\right)
$$

belongs to $\mathcal{B}(2 ; m-1)$. Hence, $R(x)=F(x) T_{q}(2 ; G)(x)$ is an element of $\mathcal{B}(1 ; m)$ and

$$
\delta R(x \mid w)=F(x) \delta T_{q}(2 ; G)(x \mid w)+\delta F(x \mid w) T_{q}(2 ; G)(x)
$$

is an element of $\mathcal{B}(1 ; m-1)$. Since

$$
f(\vec{u}) H(-i q ; \vec{u}) \psi_{l}(-i q ; \vec{u}) \quad \text { and } \quad f_{l}(\vec{u}) H(-i q ; \vec{u}) \psi_{0}(-i q ; \vec{u})
$$

belong to $L^{1}\left(\mathbb{R}^{n}\right)$ for each $l \in\{0,1, \cdots, n\}$, both of the following analytic Feynman integrals exist:

$$
E_{x}^{\operatorname{anf}_{q}}[R(x)]=\left(\prod_{j=1}^{n} \frac{-i q}{2 \pi B_{j}}\right)^{\frac{1}{2}} \int_{\mathbb{R}^{n}} f(\vec{u}) H(-i q ; \vec{u}) \psi_{0}(-i q ; \vec{u}) d \vec{u}
$$

and

$$
\begin{aligned}
E_{x}^{\operatorname{anf}_{q}}[\delta R(x \mid w)]=\left(\prod_{j=1}^{n} \frac{-i q}{2 \pi B_{j}}\right)^{\frac{1}{2}} \int_{\mathbb{R}^{n}}\left[f(\vec{u}) \sum_{l=1}^{n}\left\langle\alpha_{l}, w\right\rangle \psi_{l}(-i q ; \vec{u})\right. \\
\left.+\psi_{0}(-i q ; \vec{u}) \sum_{l=1}^{n}\left\langle\alpha_{l}, w\right\rangle f_{l}(\vec{u})\right] H(-i q ; \vec{u}) d \vec{u} .
\end{aligned}
$$

Also, for $\rho>0$ and $h>0$,

$$
\begin{aligned}
& E[|\delta R(\rho x+\rho h w \mid \rho w)|] \\
& \qquad \rho\|z\|_{a, b}\left(\prod_{j=1}^{n} 2 \pi \rho^{2} B_{j}\right)^{-\frac{1}{2}}\left[\|f\|_{2} \sum_{l=1}^{n}\left\|\psi_{l}(-i q ; \cdot)\right\|_{2}\right. \\
& \left.\quad+\left\|\psi_{0}(-i q ; \cdot)\right\|_{2} \sum_{l=1}^{n}\left\|f_{j}\right\|_{2}\right]<\infty .
\end{aligned}
$$

Hence, by Theorem 2.1, the analytic Feynman integral $E_{x}^{\operatorname{anf}_{q}}[R(x)\langle z, x\rangle]$ exists and equality (5.8) holds.

Next, choosing $G=F$ in Theorem 5.3, we obtain the following integration by parts formula.

Corollary 5.4. Let $q, F \in \mathcal{B}(2 ; m), z$, and $w$ be as in Theorem 5.3. Then

$$
\begin{aligned}
E_{x}^{\operatorname{anf}_{q}}[F(x) & \left.\delta T_{q}(2 ; F)(x \mid w)+\delta F(x \mid w) T_{q}(2 ; F)(x)\right] \\
=- & i q E_{x}^{\operatorname{anf}_{q}}\left[F(x) T_{q}(2 ; F)(x)\langle z, x\rangle\right] \\
& -(-i q)^{\frac{1}{2}}\left(z, a^{\prime}\right) E_{x}^{\operatorname{anf}_{q}}\left[F(x) T_{q}(2 ; F)(x)\right] .
\end{aligned}
$$


Our final theorem is a counterpart to Theorem 4.5 above.

Theorem 5.5. Let $q \in \mathbb{R}-\{0\}$ and let $F$ and $G$ be as in Theorem 5.3. Furthermore, assume that for each $l \in\{0,1, \cdots, n\}$,

$$
\int_{\mathbb{R}^{n}}\left|\psi_{l}(\vec{u}) H(-i q ; \vec{u})\right|^{2} d \vec{u}<\infty
$$

Let $z \in L_{a, b}^{2}[0, T]$ be given and for $t \in[0, T]$ let $w(t)=\int_{0}^{t} z(s) d b(s)$. Then

$$
\begin{gathered}
E_{x}^{\operatorname{anf}_{q}}\left[T_{q}(2 ; F)(x) \delta T_{q}(2 ; G)(x \mid w)+\delta T_{q}(2 ; F)(x \mid w) T_{q}(2 ; G)(x)\right] \\
=-i q E_{x}^{\operatorname{anf}_{q}}\left[T_{q}(2 ; F)(x) T_{q}(2 ; G)(x)\langle z, x\rangle\right] \\
\quad-(-i q)^{\frac{1}{2}}\left(z, a^{\prime}\right) E_{x}^{\operatorname{anf}_{q}}\left[T_{q}(2 ; F)(x) T_{q}(2 ; G)(x)\right] .
\end{gathered}
$$

Proof. Let $R(x)=T_{q}(2 ; F)(x) T_{q}(2 ; G)(x)$. Then $R \in \mathcal{B}(1 ; m)$ and $\delta R(\cdot \mid w) \in$ $\mathcal{B}(1 ; m-1)$. Using (5.2), (5.4), (5.9) and (5.12), we see that $E_{x}^{\operatorname{anf}_{q}}[R(x)]$ and $E_{x}^{\operatorname{anf}_{q}}[\delta R(x \mid w)]$ both exist and are given by equations $(4.22)$ and $(4,23)$ respectively. Finally, we see that (5.13) follows from Theorem 2.1 , since for $\rho>0$ and $h>0$,

$$
\begin{aligned}
& E[|\delta R(\rho x+\rho h w \mid \rho w)|] \\
& \leq \rho\|z\|_{a, b}\left(\prod_{j=1}^{n} 2 \pi \rho^{2} B_{j}\right)^{-\frac{1}{2}}\left[\left\|\phi_{0}(-i q ; \cdot)\right\|_{2} \sum_{l=1}^{n}\left\|\psi_{l}(-i q ; \cdot)\right\|_{2}\right. \\
& \left.\quad+\left\|\psi_{0}(-i q ; \cdot)\right\|_{2} \sum_{l=1}^{n}\left\|\phi_{l}(-i q ; \cdot)\right\|_{2}\right]<\infty .
\end{aligned}
$$

We finish this paper with some very brief comments about the functionals defined in Examples 4.6 and 4.7 for the case $p=2$.

We first note that for the functional $F(x) \in \mathcal{B}(2 ; m)$ defined by equation $(4.27)$ with $f\left(u_{1}\right) \in L^{2}(\mathbb{R})$ given by $(4.25)$, the $L_{2}$ analytic GFFT, $T_{-1}(2 ; F)$ does not exist because $\left|\phi_{0}\left(i ; \xi_{1}\right)\right|=+\infty$ for each $\xi_{1} \in \mathbb{R}$. In fact, the $L_{p}$ analytic GFFT, $T_{-1}(p ; F)$ does not exist for any $p \in[1,2]$.

On the other hand, it is quite easy to see that condition (5.1) holds for the function $f(\vec{u})$ given by equation (4.33). Hence, for $F(x)$ defined by equation (4.35), the $L_{2}$ analytic GFFT, $T_{-1}(2 ; F)$ exists as an element of $\mathcal{B}(2 ; m)$ and for s-a.e. $y \in C_{a, b}[0, T]$ is given by the right-hand side of equation (4.41). In fact, for all $p \in[1,2]$, the $L_{p}$ analytic GFFT, $T_{-1}(p ; F)$ exists as an element of $\mathcal{B}\left(p^{\prime} ; m\right)$ and is given by the right-hand side of equation (4.41).

\section{REFERENCES}

[1] R. H. Cameron and D. A. Storvick, An $L_{2}$ analytic Fourier-Feynman transform, Michigan Math. J. 23 (1976), 1-30. MR 53:8371

[2] - Feynman integral of variations of functions, in Gaussian random fields, Ser. Prob. Statist. 1 (1991), 144-157. MR 93b:28035

[3] K. S. Chang, B. S. Kim, and I. Yoo, Fourier-Feynman transform, convolution and first variation of functionals on abstract Wiener space, Integral transforms and Special Functions 10 (2000), 179-200. MR 2001m:28023

[4] S. J. Chang and D. L. Skoug, The effect of drift on the Fourier-Feynman transform, the convolution product and the first variation, Panamerican Math. J. 10 (2000), 25-38. 
[5] Generalized Fourier-Feynman transforms and a first variation on function space, to appear in Integral Transforms and Special Functions.

[6] T. Huffman, C. Park and D. Skoug, Analytic Fourier-Feynman transforms and convolution, Trans. Amer. Math. Soc. 347 (1995), 661-673. MR 95d:28017

[7] Generalized transforms and convolutions, Internat. J. Math. and Math. Sci. 20 (1997), 19-32. MR 97k:46047

[8] G. W. Johnson and D. L. Skoug, An $L_{p}$ Analytic Fourier-Feynman transform, Michigan Math. J. 26 (1979), 103-127. MR 81a:46050

[9] - Scale-invariant measurability in Wiener space, Pacific J. Math 83 (1979), 157-176. MR 81b:28016

[10] E. Nelson, Dynamical theories of Brownian motion (2nd edition), Math. Notes, Princeton University Press, Princeton (1967). MR 35:5001

[11] C. Park, and D. Skoug, Integration by parts formulas involving analytic Feynman integrals, Panamerican Math. J. 8 (1998), 1-11. MR 99i:46031

[12] H. L. Royden, Real Analysis (Third edition), Macmillan (1988). MR 90g:00004

[13] J. Yeh, Stochastic Processes and the Wiener Integral, Marcel Dekker, Inc., New York (1973). MR 57:14166

Department of Mathematics, Dankook University, Cheonan 330-714, Korea

E-mail address: sejchang@dankook.ac.kr

Department of Mathematics, Dankook University, Cheonan 330-714, Korea

E-mail address: jgchoi@dankook.ac.kr

Department of Mathematics and Statistics, University of Nebraska, Lincoln, NEBRASKA, 68588-0323

E-mail address: dskoug@math.unl.edu 